Yuji Liu and Shengping Chen (Guangzhou)

\title{
SOLVABILITY OF SINGULAR FRACTIONAL ORDER ELASTIC BEAM SYSTEMS WITH NONLINEARITIES DEPENDING ON LOWER DERIVATIVES
}

Abstract. In this article, existence results for positive solutions of a class of boundary-value problems for singular fractional order elastic beam systems with nonlinearities depending on lower derivatives are established. The nonlinearities in fractional differential equations may be singular at $t=0$ and $t=1$. A weighted function space is constructed and complete continuity of a nonlinear operator is proved. Our analysis relies on a well known fixed point theorem.

1. Introduction. Fractional differential equations have many applications in modeling of physical and chemical processes and in engineering, and have been of great interest recently. Moreover, mathematical aspects of fractional differential equations were discussed by many authors; see the textbooks [27], the survey papers [1, 17, papers [3, 6, 7, 8, 9, 10, 18, 24, 26, 30, 32, 33] and the references therein.

The study of coupled systems of fractional differential equations is also important, as such systems occur in various problems of applied nature; see for instance [4], [5], [11, [12], [13], [16], [19], 25].

In [4], the authors studied the solvability of the following boundary value problem for a fractional differential system:

$$
\left\{\begin{array}{l}
D_{0^{+}}^{\alpha} u(t)+f\left(t, v(t), D_{0^{+}}^{\beta-1} v(t)\right)=0, \quad t \in(0,1), 1<\alpha<2, \\
D_{0^{+}}^{\beta} u(t)+g\left(t, u(t), D_{0^{+}}^{\alpha-1} u(t)\right)=0, \quad t \in(0,1), 1<\beta<2, \\
u(0)=0, \quad u(1)=\gamma u(\eta), \quad v(0)=0, \quad v(1)=\gamma u(\eta),
\end{array}\right.
$$

2010 Mathematics Subject Classification: 26A08, 34A08, 34A34, 34A12, 45J08, 34A37, 34R15, 34B40.

Key words and phrases: positive solution, singular fractional order elastic beam system, fixed point theorem.

Received 7 November 2014; revised 21 August 2015.

Published online 9 September 2016. 
where $D_{0^{+}}^{*}$ is the Riemann-Liouville fractional derivative, $f, g:[0,1] \times \mathbb{R}^{2}$ $\rightarrow \mathbb{R}$ are continuous functions, and $\gamma \in \mathbb{R}$ and $\eta \in(0,1)$ are such that the homogeneous problem

$$
\left\{\begin{array}{l}
D_{0^{+}}^{\alpha} u(t)=0, \quad t \in(0,1), 1<\alpha<2, \\
D_{0^{+}}^{\beta} u(t)=0, \quad t \in(0,1), 1<\beta<2, \\
u(0)=0, \quad u(1)=\gamma u(\eta), \quad v(0)=0, \quad v(1)=\gamma u(\eta)
\end{array}\right.
$$

has nontrivial solutions $(u(t), v(t))=\left(c_{1} t^{\alpha-1}, c_{2} t^{\beta-1}\right)$. The methods used in [4] are based upon coincidence degree theory.

In [28], Su studied the existence of solutions of the boundary value problem

$$
\left\{\begin{array}{l}
D_{0^{+}}^{\alpha} u(t)+f\left(t, v(t), D_{0^{+}}^{p} v(t)\right)=0, \quad t \in(0,1), 1<\alpha<2, \\
D_{0^{+}}^{\beta} u(t)+g\left(t, u(t), D_{0^{+}}^{q} u(t)\right)=0, \quad t \in(0,1), 1<\beta<2, \\
u(0)=0, \quad u(1)=0, \quad v(0)=0, \quad v(1)=0,
\end{array}\right.
$$

where $f, g:[0,1] \times \mathbb{R}^{2} \rightarrow \mathbb{R}$ are continuous functions.

In [5], existence of solutions was studied for the boundary value problem

$$
\left\{\begin{array}{l}
D_{0^{+}}^{\alpha} u(t)+f\left(t, v(t), D_{0^{+}}^{p} v(t)\right)=0, \quad t \in(0,1), 1<\alpha<2, \\
D_{0^{+}}^{\beta} u(t)+g\left(t, u(t), D_{0^{+}}^{q} u(t)\right)=0, \quad t \in(0,1), 1<\beta<2, \\
u(0)=0, \quad u(1)=\gamma u(\eta), \quad v(0)=0, \quad v(1)=\gamma u(\eta),
\end{array}\right.
$$

where $1<\alpha, \beta<2, p, q, \gamma>0,0<\eta<1, \alpha-q \geq 1, \beta-p \geq 1, \gamma \eta^{\alpha-1}<1$ and $\gamma \eta^{\beta-1}<1$, and $f, g:[0,1] \times \mathbb{R} \times \mathbb{R} \rightarrow \mathbb{R}$ are continuous functions.

The main assumptions imposed on $f, g$ in [4, 5, 28] are as follows:

(BA1) there exists a nonnegative function $a \in L^{1}(0,1)$ and numbers $\epsilon_{1}, \epsilon_{2}>0, \rho_{1}, \rho_{2} \in(0,1)$ such that

$$
|f(t, x, y)| \leq a(t)+\epsilon_{1}|x|^{\rho_{1}}+\epsilon_{2}|y|^{\rho_{2}},
$$

(BA2) there exists a nonnegative function $b \in L(0,1)$ and numbers $\delta_{1}, \delta_{2}>0, \sigma_{1}, \sigma_{2} \in(0,1)$ such that

$$
|g(t, x, y)| \leq b(t)+\delta_{1}|x|^{\sigma_{1}}+\delta_{2}|y|^{\sigma_{2}} .
$$

One sees that (BA1) and (BA2) imply that both $f$ and $g$ are at most of linear growth. A natural question arises: Do BVP(I), BVP(II) and BVP(III) have solutions if (BA1) and (BA2) do not hold?

On the other hand, existence of positive solutions of fractional elastic beam equations was studied in [14, 20, 21]. To the best of our knowledge, there has been no paper discussing the solvability of singular elastic beam systems with nonlinearities depending on lower derivatives. 
With this motivation we discuss the following new boundary value problem for a singular fractional order elastic beam system:

(1)

$$
\left\{\begin{array}{l}
D_{0^{+}}^{\alpha} u(t)=f\left(t, v(t), v^{\prime}(t), v^{\prime \prime}(s), D_{0^{+}}^{n} v(t)\right), \quad t \in(0,1) \\
D_{0^{+}}^{\beta} v(t)=g\left(t, u(t), u^{\prime}(t), u^{\prime \prime}(t), D_{0^{+}}^{m} u(t)\right), \quad t \in(0,1), \\
\lim _{t \rightarrow 0} t^{4-\alpha} u(t)=0, \quad D_{0^{+}}^{\alpha-3} u(0)=0, \quad D_{0^{+}}^{\alpha-2} u(1)=0, \quad D_{0^{+}}^{\alpha-1} u(1)=0, \\
\lim _{t \rightarrow 0} t^{4-\alpha} v(t)=0, \quad D_{0^{+}}^{\alpha-3} v(0)=0, \quad D_{0^{+}}^{\beta-2} v(1)=0, \quad D_{0^{+}}^{\alpha-1} v(1)=0,
\end{array}\right.
$$

where $\alpha, \beta \in(3,4]$ and $m \in(2, \alpha-1), n \in(2, \beta-1), f, g:(0,1) \times \mathbb{R}^{4}$ $\rightarrow[0, \infty)$ are continuous, maybe singular at $t=0$ and $t=1$, and such that $|x|,|y|,|z|,|w| \leq r>0$ implies that on each subinterval $(0, c]$ with $c \in(0,1)$,

$$
\begin{aligned}
& g\left(t, t^{\alpha-2} x, t^{\alpha-3} y, t^{\alpha-4} z, t^{\alpha-m-2} w\right) \neq 0, \\
& f\left(t, t^{\beta-2} x, t^{\beta-3} y, t^{\beta-4} z, t^{\beta-m-2} w\right) \neq 0 .
\end{aligned}
$$

A couple of functions $(x, y)$ with $x, y:(0,1] \rightarrow \mathbb{R}$ is called a positive solution of $\operatorname{BVP}(1)$ if $x, x^{\prime}, x^{\prime \prime}, x^{\prime \prime \prime}, D_{0^{+}}^{\alpha} x$ and $y, y^{\prime}, y^{\prime \prime}, y^{\prime \prime \prime}, D_{0^{+}}^{\beta} y$ are in $C^{0}(0,1]$ and all equations in (1) are satisfied and $[x(t)]^{2}+[y(t)]^{2}>0$ on $(0,1)$. The purpose of this paper is to establish existence results for $\mathrm{BVP}(1)$ by using a fixed point theorem in Banach spaces.

Our methods are also useful to establish existence results for BVP(I), BVP(II) and BVP(III). We omit the details. But we point out that our assumptions imposed on $f, g$ are weaker than (BA1) and (BA2) (see Remark 3.1).

We note that fourth order two-point boundary value problems are useful for material mechanics because this kind of problems usually characterize the deflection of an elastic beam [14]. The boundary conditions are given according to the controls at the ends of the beam. For example, the nonlinear fourth order problem

$$
\left\{\begin{array}{l}
x^{(4)}(t)=\lambda f\left(t, x(t), x^{\prime}(t)\right), \quad t \in[0,1], \\
x(0)=x^{\prime}(0)=x^{\prime \prime}(1)=x^{\prime \prime \prime}(1)=0
\end{array}\right.
$$

describes the deformations of an elastic beam with one end fixed and the other free. The existence of positive solutions of various boundary value problems for elastic beam equations was extensively studied (see [2, 14, 22, 23, 29, 31]).

Few paper deals with the situation where all lower order derivatives are involved in the nonlinear term explicitly. In fact, the derivatives are of great importance in the problem in some cases, for example, in the linear elastic 
beam equation (Euler-Bernoulli equation)

$$
\left(E I u^{\prime \prime}(t)\right)^{\prime \prime}=f(t), \quad t \in(0, L),
$$

where $u(t)$ is the deformation function, $L$ is the length of the beam, $f(t)$ is the load density, $E$ is the Young modulus of elasticity and $I$ is the moment of inertia of the cross-section of the beam. In this problem, the physical meaning of the derivatives of $u(t)$ is as follows: $u^{(4)}(t)$ is the load density stiffness, $u^{\prime \prime \prime}(t)$ is the shear force stiffness, $u^{\prime \prime}(t)$ is the bending moment stiffness and $u^{\prime}(t)$ is the slope. If the payload depends on the shear force stiffness, bending moment stiffness or slope, the cases where the derivatives of the unknown function are involved in the nonlinear term $f$ explicitly occur [15]. In applications, coupled elastic beam systems occur. This leads us to study the solvability of the following BVP for coupled elastic beam equations:

$$
\begin{cases}x^{(4)}(t)=f\left(t, y(t), y^{\prime}(t), y^{\prime \prime}(t), y^{\prime \prime \prime}(t)\right), & t \in[0,1], \\ y^{(4)}(t)=g\left(t, x(t), x^{\prime}(t), x^{\prime \prime}(t), x^{\prime \prime \prime}(t)\right), & t \in[0,1], \\ x(0)=x^{\prime}(0)=x^{\prime \prime}(1)=x^{\prime \prime \prime}(1)=0, & \\ y(0)=y^{\prime}(0)=y^{\prime \prime}(1)=y^{\prime \prime \prime}(1)=0 . & \end{cases}
$$

It is easy to see that $\mathrm{BVP}(1)$ is a generalization of $\mathrm{BVP}(3)$. Hence $\mathrm{BVP}(1)$ is a fractional elastic beam system model, and our studies on BVP(1) may be useful.

The remainder of the paper is divided into two sections. In Section 2, we present some preliminary results, i.e., we construct a Banach space and prove complete continuity of a nonlinear operator. In Section 3, we establish sufficient conditions for the existence of positive solutions of $\operatorname{BVP}(1)$ by using a fixed point theorem.

2. Preliminary results. For the convenience of the readers, we present some necessary definitions from fractional calculus theory [27]. Let $\sigma_{i}>0$, $i=1,2,3$. Denote the Gamma function and Beta function, respectively, by

$$
\Gamma\left(\sigma_{1}\right)=\int_{0}^{\infty} s^{\sigma_{1}-1} e^{-s} d s, \quad \mathbf{B}\left(\sigma_{2}, \sigma_{3}\right)=\int_{0}^{1}(1-x)^{\sigma_{2}-1} x^{\sigma_{3}-1} d x .
$$

Definition 2.1. The Riemann-Liouville fractional integral of order $\alpha>0$ of a function $f:(0, \infty) \rightarrow \mathbb{R}$ is given by

$$
I_{0^{+}}^{\alpha} f(t)=\frac{1}{\Gamma(\alpha)} \int_{0}^{t}(t-s)^{\alpha-1} f(s) d s,
$$

provided that the right-hand side exists. 
Definition 2.2. The Riemann-Liouville fractional derivative of order $\alpha>0$ of a continuous function $f:(0, \infty) \rightarrow \mathbb{R}$ is given by

$$
D_{0^{+}}^{\alpha} f(t)=\frac{1}{\Gamma(n-\alpha)} \frac{d^{n}}{d t^{n}} \int_{0}^{t} \frac{f(s)}{(t-s)^{\alpha-n+1}} d s,
$$

where $n-1 \leq \alpha<n$, provided that the right-hand side exists.

One sees from [27], for $\sigma_{1}>0, \sigma_{2} \geq 0$, that

$$
I_{0^{+}}^{\sigma_{1}} t^{\sigma_{2}}=\frac{\Gamma\left(\sigma_{2}+1\right)}{\Gamma\left(\sigma_{1}+\sigma_{2}+1\right)} t^{\sigma_{1}+\sigma_{2}}, \quad D_{0^{+}}^{\sigma_{1}} t^{\sigma_{2}}=\frac{\Gamma\left(\sigma_{2}+1\right)}{\Gamma\left(\sigma_{2}-\sigma_{1}+1\right)} t^{\sigma_{2}-\sigma_{1}} .
$$

Furthermore for $n-1 \leq \alpha<n, u \in C^{0}(0,1) \cap L^{1}(0,1)$, we have

$$
I_{0+}^{\alpha} D_{0+}^{\alpha} u(t)=u(t)+C_{1} t^{\alpha-1}+C_{2} t^{\alpha-2}+\cdots+C_{n} t^{\alpha-n},
$$

where $C_{i} \in \mathbb{R}, i=1, \ldots, n[27$.

For our construction, we choose

$$
X=\left\{x:(0,1] \rightarrow \mathbb{R}: \begin{array}{l}
x, x^{\prime}, x^{\prime \prime}, D_{0^{+}}^{m} x \in C^{0}(0,1] \\
\text { and the limits } \\
\lim _{t \rightarrow 0} t^{2-\alpha} x(t), \lim _{t \rightarrow 0} t^{3-\alpha} x^{\prime}(t), \\
\lim _{t \rightarrow 0} t^{4-\alpha} x^{\prime \prime}(t), \lim _{t \rightarrow 0} t^{2+m-\alpha} D_{0^{+}}^{m} x(t) \text { exist }
\end{array}\right\}
$$

with the norm

$$
\begin{aligned}
&\|u\|_{X}=\max \left\{\sup _{t \in(0,1]} t^{2-\alpha}|u(t)|, \sup _{t \in(0,1]} t^{3-\alpha}\left|u^{\prime}(t)\right|,\right. \\
&\left.\sup _{t \in(0,1]} t^{4-\alpha}\left|u^{\prime \prime}(t)\right|, \sup _{t \in(0,1]} t^{2+m-\alpha}\left|D_{0^{+}}^{m} u(t)\right|\right\}, \quad u \in X .
\end{aligned}
$$

Lemma 2.0. Suppose that $\alpha \in(3,4)$ and $m \in(2, \alpha-1)$. Then $X$ is a real Banach space.

Proof. It is easy to see that $X$ is a normed linear space. Let $\left\{x_{u}\right\}$ be a Cauchy sequence in $X$. Then $\left\|x_{u}-x_{v}\right\| \rightarrow 0$ as $u, v \rightarrow \infty$. It follows that

$$
\begin{aligned}
& \sup _{t \in(0,1]} t^{2-\alpha}\left|x_{u}(t)-x_{v}(t)\right| \rightarrow 0, \\
& \sup _{t \in(0,1]} t^{3-\alpha}\left|x_{u}^{\prime}(t)-x_{v}^{\prime}(t)\right| \rightarrow 0, \\
& \sup _{t \in(0,1]} t^{4-\alpha}\left|x_{u}^{\prime \prime}(t)-x_{v}^{\prime \prime}(t)\right| \rightarrow 0, \\
& \sup _{t \in(0,1]} t^{2+m-\alpha}\left|D_{0^{+}}^{m} x_{u}(t)-D_{0^{+}}^{m} x_{v}(t)\right| \rightarrow 0, \quad u, v \rightarrow \infty .
\end{aligned}
$$


Define

$$
\begin{aligned}
t^{2-\alpha} \bar{x}(t) & = \begin{cases}\lim _{t \rightarrow 0^{+}} t^{2-\alpha} x(t), & t=0, \\
t^{2-\alpha} x(t), & t \in(0,1],\end{cases} \\
t^{3-\alpha} \bar{x}^{\prime}(t) & = \begin{cases}\lim _{t \rightarrow 0^{+}} t^{3-\alpha} x^{\prime}(t), & t=0, \\
t^{3-\alpha} x^{\prime}(t), & t \in(0,1],\end{cases} \\
t^{4-\alpha} \bar{x}^{\prime \prime}(t) & = \begin{cases}\lim _{t \rightarrow 0^{+}} t^{4-\alpha} x^{\prime \prime}(t), & t=0, \\
t^{4-\alpha} x^{\prime \prime}(t), & t \in(0,1],\end{cases} \\
t^{2+m-\alpha} D_{0^{+}}^{m} \bar{x}(t) & = \begin{cases}\lim _{t \rightarrow 0^{+}} t^{2+m-\alpha} D_{0^{+}}^{m} x(t), & t=0, \\
t^{2+m-\alpha} D_{0^{+}}^{m} x(t), & t \in(0,1] .\end{cases}
\end{aligned}
$$

We know that

$$
t \mapsto t^{2-\alpha} \bar{x}(t), \quad t \mapsto t^{3-\alpha} \bar{x}^{\prime}(t), \quad t \mapsto t^{4-\alpha} \bar{x}^{\prime \prime}(t), \quad t \mapsto t^{2+m-\alpha} D_{0^{+}}^{m} \bar{x}(t)
$$

are continuous on $[0,1]$. Thus

$$
t \mapsto t^{2-\alpha} \bar{x}_{u}(t), \quad t \mapsto t^{3-\alpha} \bar{x}_{u}^{\prime}(t), \quad t \mapsto t^{4-\alpha} \bar{x}_{u}^{\prime \prime}(t), \quad t \mapsto t^{2+m-\alpha} D_{0^{+}}^{m} \bar{x}_{u}(t)
$$

are Cauchy sequences in $C[0,1]$. Hence as $u \rightarrow \infty, t^{2-\alpha} \bar{x}_{u}(t)$ uniformly converges to some $x_{0} \in C[0,1], t^{3-\alpha} \bar{x}_{u}^{\prime}(t)$ uniformly converges to some $y_{0} \in C[0,1], t^{4-\alpha} \bar{x}_{u}^{\prime \prime}(t)$ uniformly converges to some $z_{0} \in C[0,1]$, and $t^{2+m-\alpha} D_{0^{+}}^{m} \bar{x}_{u}(t)$ uniformly converges to some $w_{0} \in C[0,1]$.

For some $c_{u} \in \mathbb{R}$ we have

$$
\begin{aligned}
\mid \bar{x}_{u}(t)-c_{u} & -\int_{0}^{t} s^{\alpha-3} y_{0}(s) d s\left|\leq \int_{0}^{t}\right| \bar{x}_{u}^{\prime}(s)-s^{\alpha-3} y_{0}(s) \mid d s \\
& =\int_{0}^{t} s^{\alpha-3}\left|s^{3-\alpha} \bar{x}_{u}^{\prime}(s)-y_{0}(s)\right| d s \leq \int_{0}^{t} s^{\alpha-3} d s \sup _{t \in[0,1]}\left|t^{3-\alpha} \bar{x}_{u}^{\prime}(t)-y_{0}(t)\right| \\
& =\frac{t^{\alpha-2}}{\alpha-2} \sup _{t \in[0,1]}\left|t^{3-\alpha} \bar{x}_{u}^{\prime}(t)-y_{0}(t)\right| \leq \sup _{t \in[0,1]}\left|t^{3-\alpha} \bar{x}_{u}^{\prime}(t)-y_{0}(t)\right| \\
& \rightarrow 0 \quad \text { uniformly as } n \rightarrow \infty .
\end{aligned}
$$

So $\lim _{u \rightarrow \infty}\left(\bar{x}_{u}(t)-c_{u}\right)=\int_{0}^{t} s^{\alpha-3} y_{0}(s) d s$. It follows that $t^{2-\alpha} x_{0}(t)-c_{0}=$ $\int_{0}^{t} s^{\alpha-3} y_{0}(s) d s$. Thus $y_{0}(t)=t^{3-\alpha}\left[t^{2-\alpha} x_{0}(t)\right]^{\prime}$.

For some $c_{u}, d_{u} \in \mathbb{R}$ we have

$$
\begin{aligned}
& \left|\bar{x}_{u}(t)-c_{u}-d_{u} t-\int_{0}^{t}(t-s) s^{\alpha-4} z_{0}(s) d s\right| \\
& =\left|\int_{0}^{t}(t-s)\left[\bar{x}_{u}^{\prime \prime}(s) d s-s^{\alpha-4} z_{0}(s)\right] d s\right|=\int_{0}^{t}(t-s) s^{\alpha-4}\left|s^{4-\alpha} \bar{x}_{u}^{\prime \prime}(s)-z_{0}(s)\right| d s \\
& \leq \int_{0}^{t}(t-s) s^{\alpha-4} d s \sup _{t \in[0,1]} \mid t^{4-\alpha} \bar{x}_{u}^{\prime \prime}(t)-z_{0}(t \mid
\end{aligned}
$$




$$
\begin{aligned}
& =t^{\alpha-2} \int_{0}^{1}(1-w) w^{\alpha-4} d w \sup _{t \in[0,1]}\left|t^{4-\alpha} \bar{x}_{u}^{\prime \prime}(t)-z_{0}(t)\right| \leq \sup _{t \in[0,1]}\left|t^{4-\alpha} x_{n}^{\prime \prime}(t)-z_{0}(t)\right| \\
& =t^{\alpha-2} \mathbf{B}(2, \alpha-3) \sup _{t \in[0,1]}\left|t^{4-\alpha} \bar{x}_{u}^{\prime \prime}(t)-z_{0}(t)\right| \leq \sup _{t \in[0,1]}\left|t^{4-\alpha} \bar{x}_{u}^{\prime \prime}(t)-z_{0}(t)\right| \rightarrow 0
\end{aligned}
$$

uniformly as $u \rightarrow \infty$. So $\lim _{u \rightarrow \infty}\left(\bar{x}_{u}(t)-c_{u}-d_{u} t\right)=\int_{0}^{t}(t-s) s^{\alpha-4} z_{0}(s) d s$. It follows that $t^{2-\alpha} x_{0}(t)-c_{0}-d_{0} t=\int_{0}^{t}(t-s) s^{\alpha-4} z_{0}(s) d s$. Thus $z_{0}(t)=$ $t^{4-\alpha}\left[t^{2-\alpha} x_{0}(t)\right]^{\prime \prime}$.

For some $c_{u}, d_{u}, e_{u} \in \mathbb{R}$ we have

$$
\begin{aligned}
&\left|\bar{x}_{u}(t)+c_{u} t^{m-1}+d_{u} t^{m-2}+e_{u} t^{m-3}-I_{0^{+}}^{m} t^{\alpha-m-2} w_{0}(t)\right| \\
&=\left|I_{0^{+}}^{m} D_{0^{+}}^{m} \bar{x}_{u}(t)--I_{0^{+}}^{m} t^{\alpha-m-2} w_{0}(t)\right| \\
&=\left|\int_{0}^{t} \frac{(t-s)^{m-1}}{\Gamma(m)} D_{0^{+}}^{m} \bar{x}_{u}(s) d s-\int_{0}^{t} \frac{(t-s)^{m-1}}{\Gamma(m)} s^{\alpha-m-2} w_{0}(s) d s\right| \\
& \leq \int_{0}^{t} \frac{(t-s)^{m-1}}{\Gamma(m)}\left|D_{0^{+}}^{m} \bar{x}_{u}(s)-s^{\alpha-m-2} w_{0}(s)\right| d s \\
& \leq \int_{0}^{t} \frac{(t-s)^{m-1}}{\Gamma(m)} s^{\alpha-m-2}\left|s^{2+m-\alpha} D_{0^{+}}^{m} \bar{x}_{u}(s)-w_{0}(s)\right| d s \\
& \leq \int_{0}^{t} \frac{(t-s)^{m-1}}{\Gamma(m)} s^{\alpha-m-2} d s \sup _{t \in[0,1]}\left|t^{2+m-\alpha} D_{0^{+}}^{m} \bar{x}_{u}(t)-w_{0}(t)\right| \\
&=t^{\alpha-2} \int_{0}^{1} \frac{(1-w)^{m-1}}{\Gamma(m)} w^{\alpha-m-2} d w \sup _{t \in[0,1]}\left|t^{2+m-\alpha} D_{0^{+}}^{m} \bar{x}_{u}(t)-w_{0}(t)\right| \\
&=t^{\alpha-2} \frac{\mathbf{B}(m, \alpha-m-1)}{\Gamma(m)} \sup _{t \in[0,1]}\left|t^{2+m-\alpha} D_{0^{+}}^{m} \bar{x}_{u}(t)-w_{0}(t)\right| \rightarrow 0
\end{aligned}
$$

uniformly as $u \rightarrow \infty$. So $\lim _{u \rightarrow \infty}\left(\bar{x}_{u}(t)+c_{u} t^{m-1}+d_{u} t^{m-2}+e_{u} t^{m-3}\right)=$ $I_{0^{+}}^{\beta} t^{\alpha-m-2} w_{0}(t)$. It follows that $\left.t^{2-\alpha} x_{0}(t)+c_{0} t^{m-1}+d_{0} t^{m-2}+e_{0} t^{m-3}\right)=$ $I_{0^{+}}^{\beta} t^{\alpha-m-2} w_{0}(t)$. Thus $w_{0}(t)=t^{2+m-\alpha} D_{0^{+}}^{m}\left[t^{2-\alpha} x_{0}(t)\right]$.

So $x_{n} \rightarrow x_{0}$ as $n \rightarrow \infty$ in $X$ and $t^{2-\alpha} x_{0} \in X$. It follows that $X$ is a Banach space.

Choose

$$
Y=\left\{y:(0,1] \rightarrow \mathbb{R}: \quad \begin{array}{l}
y, y^{\prime}, y^{\prime \prime}, D_{0^{+}}^{n} y \in C^{0}(0,1] \\
\text { and the limits } \\
\lim _{t \rightarrow 0} t^{2-\beta} y(t), \lim _{t \rightarrow 0} t^{3-\beta} y^{\prime}(t), \\
\lim _{t \rightarrow 0} t^{4-\beta} y^{\prime \prime}(t), \lim _{t \rightarrow 0} t^{2+n-\beta} y^{\prime \prime \prime}(t) \text { exist }
\end{array}\right\}
$$


with the norm

$$
\begin{aligned}
\|v\|_{Y}=\max \left\{\sup _{t \in(0,1]} t^{2-\beta}|v(t)|, \sup _{t \in(0,1]} t^{3-\beta}\left|v^{\prime}(t)\right|,\right. & \\
\left.\sup _{t \in(0,1]} t^{4-\beta}\left|v^{\prime \prime}(t)\right|, \sup _{t \in(0,1]} t^{2+n-\beta}\left|v^{\prime \prime \prime}(t)\right|\right\}, & v \in Y .
\end{aligned}
$$

One can show similarly that $Y$ is a real Banach space.

Thus, $(X \times Y,\|\cdot\|)$ is Banach space with the norm defined by

$$
\|(x, y)\|=\max \left\{\|x\|=\|x\|_{X},\|y\|=\|y\|_{Y}\right\} \quad \text { for }(x, y) \in X \times Y .
$$

LEMMA 2.1. Suppose that

(B0) $h \in C^{0}(0,1)$ and there exist $M>0, k>-1$ and $\sigma \leq 0$ such that $2+\sigma+k>0$ and $|h(t)| \leq M t^{k}(1-t)^{\sigma}$ for all $t \in(0,1)$.

Then $x \in X$ is a solution of

$$
\left\{\begin{array}{l}
D^{\alpha} x(t)=h(t), \quad 0<t<1 \\
\lim _{t \rightarrow 0} t^{4-\alpha} x(t)=0, \lim _{t \rightarrow 0} D_{0^{+}}^{\alpha-3} x(t)=0, D_{0^{+}}^{\alpha-2} x(1)=0, D_{0^{+}}^{\alpha-1} x(1)=0
\end{array}\right.
$$

if and only if $x$ satisfies

$$
\begin{aligned}
x(t)= & \int_{0}^{t} \frac{(t-s)^{\alpha-1}}{\Gamma(\alpha)} h(s) d s-\left[\frac{t^{\alpha-1}}{\Gamma(\alpha)}-\frac{t^{\alpha-2}}{\Gamma(\alpha-1)}\right] \int_{0}^{1} h(s) d s \\
& -\frac{t^{\alpha-2}}{\Gamma(\alpha-1)} \int_{0}^{1}(1-s) h(s) d s=\int_{0}^{1} G(t, s) h(s) d s,
\end{aligned}
$$

where $G$ is defined by

$$
G(t, s)= \begin{cases}-\frac{t^{\alpha-2}(1-s)}{\Gamma(\alpha-1)}-\frac{t^{\alpha-1}}{\Gamma(\alpha)}+\frac{t^{\alpha-2}}{\Gamma(\alpha-1)}+\frac{(t-s)^{\alpha-1}}{\Gamma(\alpha)}, & s \leq t \\ -\frac{t^{\alpha-2}(1-s)}{\Gamma(\alpha-1)}-\frac{t^{\alpha-1}}{\Gamma(\alpha)}+\frac{t^{\alpha-2}}{\Gamma(\alpha-1)}, & s>t .\end{cases}
$$

Proof. Since $h \in C^{0}(0,1)$ and there exist $M>0, k>-1$ and $\sigma \in$ $(-2-k, 0]$ such that $|h(t)| \leq M t^{k}(1-t)^{\sigma}$ for all $t \in(0,1)$, we have

$$
\begin{aligned}
\left|\int_{0}^{t} \frac{(t-s)^{\alpha-1}}{\Gamma(\alpha)} h(s) d s\right| \leq \int_{0}^{t} \frac{(t-s)^{\alpha-1}}{\Gamma(\alpha)} M s^{k}(1-s)^{\sigma} d s \\
\quad \leq M \int_{0}^{t} \frac{(t-s)^{\alpha+\sigma-1}}{\Gamma(\alpha)} s^{k} d s=M t^{\alpha+\sigma+k} \int_{0}^{1} \frac{(1-w)^{\alpha+\sigma-1}}{\Gamma(\alpha)} w^{k} d w \\
\quad=M t^{\alpha+\sigma+k \frac{\mathbf{B}(\alpha+\sigma, k+1)}{\Gamma(\alpha)}}
\end{aligned}
$$




$$
\begin{aligned}
& \left|\int_{0}^{t} \frac{(t-s)^{\alpha-2}}{\Gamma(\alpha-1)} h(s) d s\right| \leq M t^{\alpha+\sigma+k-1} \frac{\mathbf{B}(\alpha+\sigma-1, k+1)}{\Gamma(\alpha-1)}, \\
& \left|\int_{0}^{t} \frac{(t-s)^{\alpha-3}}{\Gamma(\alpha-2)} h(s) d s\right| \leq M t^{\alpha+\sigma+k-2} \frac{\mathbf{B}(\alpha+\sigma-2, k+1)}{\Gamma(\alpha-2)}, \\
& \left|\int_{0}^{t} \frac{(t-s)^{\alpha-4}}{\Gamma(\alpha-3)} h(s) d s\right| \leq M t^{\alpha+\sigma+k-3} \frac{\mathbf{B}(\alpha+\sigma-3, k+1)}{\Gamma(\alpha-3)}, \\
& \left|\int_{0}^{t}(t-s) h(s) d s\right| \leq \int_{0}^{t}(t-s) M s^{k}(1-s)^{\sigma} d s \\
& \leq M \int_{0}^{t}(t-s)^{1+\sigma} s^{k} d s=M t^{2+\sigma+k} \int_{0}^{1}(1-w)^{1+\sigma} w^{k} d w \\
& =M t^{2+\sigma+k} \mathbf{B}(2+\sigma, k+1) \text {, } \\
& \left|\int_{0}^{t} h(s) d s\right| \leq M t^{1+\sigma+k} \mathbf{B}(1+\sigma, k+1) \text {. }
\end{aligned}
$$

So the integrals

$$
\begin{aligned}
\int_{0}^{t} \frac{(t-s)^{\alpha-1}}{\Gamma(\alpha)} & h(s) d s, \int_{0}^{t} \frac{(t-s)^{\alpha-2}}{\Gamma(\alpha-1)} h(s) d s, \int_{0}^{t} \frac{(t-s)^{\alpha-3}}{\Gamma(\alpha-2)} h(s) d s \\
& \int_{0}^{t} \frac{(t-s)^{\alpha-4}}{\Gamma(\alpha-3)} h(s) d s \text { and } \int_{0}^{t}(t-s) h(s) d s, \int_{0}^{t} h(s) d s, \quad t \in(0,1]
\end{aligned}
$$

are convergent.

For $t \in(0,1], D_{0^{+}}^{\alpha} u(t)=-h(t)$ implies that there exist constants $c_{i}$ $(i=1,2,3,4)$ such that

$$
x(t)=\int_{0}^{t} \frac{(t-s)^{\alpha-1}}{\Gamma(\alpha)} h(s) d s+c_{1} t^{\alpha-1}+c_{2} t^{\alpha-2}+c_{3} t^{\alpha-3}+c_{4} t^{\alpha-4}
$$

with

$$
\begin{aligned}
D_{0^{+}}^{\alpha-3} x(t) & =\int_{0}^{t} \frac{(t-s)^{2}}{2} h(s) d s+c_{1} \frac{\Gamma(\alpha)}{2} t^{2}+c_{2} \Gamma(\alpha-1) t+c_{3} \Gamma(\alpha-2), \\
D_{0^{+}}^{\alpha-2} x(t) & =\int_{0}^{t}(t-s) h(s) d s+c_{1} \Gamma(\alpha) t+c_{2} \Gamma(\alpha-1), \\
D_{0^{+}}^{\alpha-1} x(t) & =\int_{0}^{t} h(s) d s+c_{1} \Gamma(\alpha) .
\end{aligned}
$$


One sees that

$$
\begin{aligned}
t^{4-\alpha} \int_{0}^{t} & \frac{(t-s)^{\alpha-1}}{\Gamma(\alpha)} h(s) d s \rightarrow 0 \quad \text { as } t \rightarrow 0(\text { note } 4+\sigma+k>0) \\
& \int_{0}^{t}(t-s)^{2} h(s) d s \rightarrow 0 \quad \text { as } t \rightarrow 0(\text { note } 2+\sigma+k>0) .
\end{aligned}
$$

Now, (6) and (7) with $\lim _{t \rightarrow 0} t^{4-\alpha} x(t)=0$ imply that $c_{4}=0$.

Furthermore, (8) with $\lim _{t \rightarrow 0} D_{0^{+}}^{\alpha-3} x(t)=0$ implies $c_{3}=0$.

Using $D_{0^{+}}^{\alpha-2} x(1)=0$ and $D_{0^{+}}^{\alpha-1} x(1)=0$, we get

$$
c_{1} \Gamma(\alpha)+c_{2} \Gamma(\alpha-1)=-\int_{0}^{1}(1-s) h(s) d s, \quad c_{1} \Gamma(\alpha)=-\int_{0}^{1} h(s) d s .
$$

It follows that

$$
\begin{aligned}
& c_{1}=-\frac{1}{\Gamma(\alpha)} \int_{0}^{1} h(s) d s \\
& c_{2}=\frac{1}{\Gamma(\alpha-1)}\left[-\int_{0}^{1}(1-s) h(s) d s+\int_{0}^{1} h(s) d s\right] .
\end{aligned}
$$

Then

$$
\begin{aligned}
x(t)=\int_{0}^{t} \frac{(t-s)^{\alpha-1}}{\Gamma(\alpha)} h(s) d s- & {\left[\frac{t^{\alpha-1}}{\Gamma(\alpha)}-\frac{t^{\alpha-2}}{\Gamma(\alpha-1)}\right] \int_{0}^{1} h(s) d s } \\
& \quad-\frac{t^{\alpha-2}}{\Gamma(\alpha-1)} \int_{0}^{1}(1-s) h(s) d s=\int_{0}^{1} G(t, s) h(s) d s,
\end{aligned}
$$

where $G$ is defined above. Hence $x$ satisfies (5).

Conversely, if $x$ satisfies (5), then

$$
\begin{aligned}
x^{\prime}(t)= & -\int_{0}^{t} \frac{(t-s)^{\alpha-2}}{\Gamma(\alpha-1)} h(s) d s+\left[\frac{t^{\alpha-2}}{\Gamma(\alpha-1)}-\frac{t^{\alpha-3}}{\Gamma(\alpha-2)}\right] \int_{0}^{1} h(s) d s \\
& +\frac{t^{\alpha-3}}{\Gamma(\alpha-2)} \int_{0}^{1}(1-s) h(s) d s \\
x^{\prime \prime}(t)= & -\int_{0}^{t} \frac{(t-s)^{\alpha-3}}{\Gamma(\alpha-2)} h(s) d s+\left[\frac{t^{\alpha-3}}{\Gamma(\alpha-2)}-\frac{t^{\alpha-4}}{\Gamma(\alpha-3)}\right] \int_{0}^{1} h(s) d s \\
& +\frac{t^{\alpha-4}}{\Gamma(\alpha-3)} \int_{0}^{1}(1-s) h(s) d s,
\end{aligned}
$$




$$
\begin{aligned}
D_{0^{+}}^{m} x(t)= & -\int_{0}^{t} \frac{(t-s)^{\alpha-m-1}}{\Gamma(\alpha-m)} h(s) d s \\
& +\left[\frac{t^{\alpha-m-1}}{\Gamma(\alpha-m)}-\frac{t^{\alpha-m-2}}{\Gamma(\alpha-m-1)}\right] \int_{0}^{1} h(s) d s \\
& +\frac{t^{\alpha-m-2}}{\Gamma(\alpha-m-1)} \int_{0}^{1}(1-s) h(s) d s .
\end{aligned}
$$

It is easy to see that $x, x^{\prime}, x^{\prime \prime}, x^{\prime \prime \prime} \in C^{0}(0,1]$ and

$$
\begin{aligned}
& t^{2-\alpha}\left|\int_{0}^{t} \frac{(t-s)^{\alpha-1}}{\Gamma(\alpha)} h(s) d s\right| \leq M t^{2-\alpha} t^{\alpha+\sigma+k} \frac{\mathbf{B}(\alpha+\sigma, k+1)}{\Gamma(\alpha)} \\
& t^{3-\alpha}\left|\int_{0}^{t} \frac{(t-s)^{\alpha-2}}{\Gamma(\alpha-1)} h(s) d s\right| \leq M t^{3-\alpha} t^{\alpha+\sigma+k-1} \frac{\mathbf{B}(\alpha+\sigma-1, k+1)}{\Gamma(\alpha-1)}, \\
& t^{4-\alpha}\left|\int_{0}^{t} \frac{(t-s)^{\alpha-3}}{\Gamma(\alpha-2)} h(s) d s\right| \leq M t^{4-\alpha} t^{\alpha+\sigma+k-2} \frac{\mathbf{B}(\alpha+\sigma-2, k+1)}{\Gamma(\alpha-2)}, \\
& t^{2+m-\alpha}\left|\int_{0}^{t} \frac{(t-s)^{\alpha-m-1}}{\Gamma(\alpha-m)} h(s) d s\right| \leq M t^{2+m-\alpha} t^{\alpha+\sigma+k-m} \frac{\mathbf{B}(\alpha+\sigma-m, k+1)}{\Gamma(\alpha-3)} .
\end{aligned}
$$

So

$$
\lim _{t \rightarrow 0} t^{2-\alpha} x(t), \quad \lim _{t \rightarrow 0} t^{3-\alpha} x^{\prime}(t), \quad \lim _{t \rightarrow 0} t^{4-\alpha} x^{\prime \prime}(t), \quad \lim _{t \rightarrow 0} t^{2+m-\alpha} D_{0^{+}}^{m} x(t)
$$

exist. We can show that $x \in X$ and $x$ is a solution of $\operatorname{BVP}(4)$.

So $x \in X$ is a solution of problem (4) if and only if $x$ satisfies (5). The proof is complete.

REMark 2.1. $G(t, s) \geq 0$ for all $(t, s) \in[0,1] \times[0,1]$. In fact, for $s>t$, we have

$$
\begin{array}{r}
-\frac{t^{\alpha-2}(1-s)}{\Gamma(\alpha-1)}-\frac{t^{\alpha-1}}{\Gamma(\alpha)}+\frac{t^{\alpha-2}}{\Gamma(\alpha-1)}=\frac{t^{\alpha-2}}{\Gamma(\alpha)}(-(\alpha-1)(1-s)-t+(\alpha-1)) \\
=(\alpha-1) s-t>(\alpha-1) s-s=(\alpha-2) s>0 .
\end{array}
$$

For $0 \leq s \leq t$, set

$$
\phi(s)=-\frac{t^{\alpha-2}(1-s)}{\Gamma(\alpha-1)}-\frac{t^{\alpha-1}}{\Gamma(\alpha)}+\frac{t^{\alpha-2}}{\Gamma(\alpha-1)}+\frac{(t-s)^{\alpha-1}}{\Gamma(\alpha)}, \quad s \leq t .
$$

One finds $\phi(0)=0$ and

$$
\phi^{\prime}(s)=\frac{t^{\alpha-2}}{\Gamma(\alpha-1)}-\frac{(t-s)^{\alpha-2}}{\Gamma(\alpha-1)} \geq 0 .
$$


Hence $\phi(s) \geq 0$ for $s \in[0, t]$. From the above discussion, we have $G(t, s) \geq 0$ for all $(t, s) \in[0,1] \times[0,1]$.

REMARK 2.2. If $h$ is nonnegative on $(0,1)$ and $h(t) \not \equiv 0$ on each subinterval $(0, c]$ with $c \in(0,1)$, then $x(t)$ is positive on $(0,1)$. In fact, by Remark 2.1, $x(t) \geq 0$ on $(0,1)$. Suppose that there exists $t_{0} \in(0,1)$ such that $x\left(t_{0}\right)=0$. From $-\frac{t^{\alpha-2}(1-s)}{\Gamma(\alpha-1)}-\frac{t^{\alpha-1}}{\Gamma(\alpha)}+\frac{t^{\alpha-2}}{\Gamma(\alpha-1)} \geq 0$ for every $t, s \in[0,1]$, we have

$$
\begin{aligned}
0=x\left(t_{0}\right)= & \int_{0}^{t_{0}} \frac{\left(t_{0}-s\right)^{\alpha-1}}{\Gamma(\alpha)} h(s) d s-\left[\frac{t_{0}^{\alpha-1}}{\Gamma(\alpha)}-\frac{t_{0}^{\alpha-2}}{\Gamma(\alpha-1)}\right] \int_{0}^{1} h(s) d s \\
& -\frac{t_{0}^{\alpha-2}}{\Gamma(\alpha-1)} \int_{0}^{1}(1-s) h(s) d s \\
\geq & \int_{0}^{t_{0}} \frac{\left(t_{0}-s\right)^{\alpha-1}}{\Gamma(\alpha)} h(s) d s \geq 0 .
\end{aligned}
$$

As $h \in C^{0}(0,1)$ and $h$ is nonnegative, we have $h(t) \equiv 0$ on $\left(0, t_{0}\right)$, which is a contradiction. Hence $x$ is positive on $(0,1)$.

Lemma 2.2. Suppose that (B0) holds. Then $y \in Y$ is a solution of

$$
\left\{\begin{array}{l}
D^{\beta} y(t)=h(t), \quad 0<t<1 \\
\lim _{t \rightarrow 0} t^{4-\alpha} y(t)=0, \lim _{t \rightarrow 0} D_{0^{+}}^{\alpha-3} y(t)=0, D_{0^{+}}^{\beta-2} y(1)=0, D_{0^{+}}^{\alpha-1} y(1)=0
\end{array}\right.
$$

if and only if $y$ satisfies

$$
\begin{aligned}
y(t)=\int_{0}^{t} \frac{(t-s)^{\beta-1}}{\Gamma(\beta)} h(s) d s-\left[\frac{t^{\beta-1}}{\Gamma(\beta)}-\frac{t^{\beta-2}}{\Gamma(\beta-1)}\right] \int_{0}^{1} h(s) d s \\
-\frac{t^{\beta-2}}{\Gamma(\beta-1)} \int_{0}^{1}(1-s) h(s) d s=\int_{0}^{1} H(t, s) h(s) d s,
\end{aligned}
$$

where $H$ is defined by

$$
H(t, s)= \begin{cases}-\frac{t^{\beta-2}(1-s)}{\Gamma(\beta-1)}-\frac{t^{\beta-1}}{\Gamma(\beta)}+\frac{t^{\beta-2}}{\Gamma(\beta-1)}+\frac{(t-s)^{\beta-1}}{\Gamma(\beta)}, & s \leq t, \\ -\frac{t^{\beta-2}(1-s)}{\Gamma(\beta-1)}-\frac{t^{\beta-1}}{\Gamma(\beta)}+\frac{t^{\beta-2}}{\Gamma(\beta-1)}, & s>t .\end{cases}
$$

Proof. The proof is similar to that of Lemma 2.1 and is omitted.

REMARK 2.3. Similarly to Remark 2.1 , we can prove that $H(t, s) \geq 0$ for all $(t, s) \in[0,1] \times[0,1]$.

REMARK 2.4. If $h$ is nonnegative on $(0,1)$ and $h(t) \not \equiv 0$ on each subinterval $(0, c]$ with $c \in(0,1)$, then $y(t)$ is positive on $(0,1)$. 
Define an operator $T$ on $X \times Y$, for $(x, y) \in X \times Y$, by $T(x, y)(t)=$ $\left(\left(T_{1} y\right)(t),\left(T_{2} x\right)(t)\right)$ with

$$
\begin{aligned}
\left(T_{1} x\right)(t)= & \int_{0}^{t} \frac{(t-s)^{\alpha-1}}{\Gamma(\alpha)} f\left(s, y(s), y^{\prime}(s), y^{\prime \prime}(s), D_{0^{+}}^{n} y(s)\right) d s \\
& -\left[\frac{t^{\alpha-1}}{\Gamma(\alpha)}-\frac{t^{\alpha-2}}{\Gamma(\alpha-1)}\right] \int_{0}^{1} f\left(s, y(s), y^{\prime}(s), y^{\prime \prime}(s), D_{0^{+}}^{n} y(s)\right) d s \\
& -\frac{t^{\alpha-2}}{\Gamma(\alpha-1)} \int_{0}^{1}(1-s) f\left(s, y(s), y^{\prime}(s), y^{\prime \prime}(s), D_{0^{+}}^{n} y(s)\right) d s
\end{aligned}
$$

and

$$
\begin{aligned}
\left(T_{2} y\right)(t)= & \int_{0}^{t} \frac{(t-s)^{\beta-1}}{\Gamma(\beta)} g\left(s, x(s), x^{\prime}(s), x^{\prime \prime}(s), D_{0^{+}}^{m} x(s)\right) d s \\
& -\left[\frac{t^{\beta-1}}{\Gamma(\beta)}-\frac{t^{\beta-2}}{\Gamma(\beta-1)}\right] \int_{0}^{1} g\left(s, x(s), x^{\prime}(s), x^{\prime \prime}(s), D_{0^{+}}^{m} x(s)\right) d s \\
& -\frac{t^{\beta-2}}{\Gamma(\beta-1)} \int_{0}^{1}(1-s) g\left(s, x(s), x^{\prime}(s), x^{\prime \prime}(s), D_{0^{+}}^{m} x(s)\right) d s .
\end{aligned}
$$

REMARK 2.5. If both $f, g$ are nonnegative, and

$$
\begin{aligned}
& f\left(t, t^{\beta-2} x, t^{\beta-3} y, t^{\beta-4} z, t^{\beta-n-2} w\right) \not \equiv 0, \\
& g\left(t, t^{\alpha-2} x, t^{\alpha-3} y, t^{\alpha-4} z, t^{\alpha-n-2} w\right) \not \equiv 0
\end{aligned}
$$

on each subinterval $(0, c]$ with $c \in(0,1)$, we know from Lemmas 2.1 and 2.2 and Remarks 2.1-2.4 that $(x, y) \in X \times Y$ is a positive solution of $\operatorname{BVP}(1)$ if and only if $(x, y)$ is a fixed point of $T$.

Lemma 2.3. Suppose that

(B1) $f(t, x, y, z, w)$ is continuous on $(0,1) \times \mathbb{R}^{4}$ and for each $r>0$ there exist $M_{r}>0, k_{1}>-1, \sigma_{1} \leq 0$ such that $2+\sigma_{1}+k_{1}>0$ and

$$
\left|f\left(t, t^{\beta-2} x, t^{\beta-3} y, t^{\beta-4} z, t^{\beta-n-2} w\right)\right| \leq M_{r} t^{k_{1}}(1-t)^{\sigma_{1}}
$$

for all $t \in(0,1)$ and $|x|,|y|,|z|,|w| \leq r$.

Then $T_{1}: Y \rightarrow X$ is completely continuous.

Proof. We divide the proof into four steps.

Step 1. $T_{1}: Y \rightarrow X$ is well defined. 
For $y \in Y$, there exists $r>0$ such that

$$
\begin{aligned}
\max \left\{\sup _{t \in(0,1]} t^{2-\beta}|y(t)|,\right. & \sup _{t \in(0,1]} t^{3-\beta}\left|y^{\prime}(t)\right|, \\
& \left.\sup _{t \in(0,1]} t^{4-\beta}\left|y^{\prime \prime}(t)\right|, \sup _{t \in(0,1]} t^{2+n-\beta}\left|D_{0^{+}}^{n} y(t)\right|\right\}<r .
\end{aligned}
$$

Then there exist $k_{1}>-1, \sigma_{1} \leq 0$ and $M_{r} \geq 0$ such that $2+k_{1}+\sigma_{1}>0$ and

$$
\begin{aligned}
& \left|f\left(t, y(t), y^{\prime}(t), y^{\prime \prime}(t), D_{0^{+}}^{n} y(t)\right)\right| \\
= & \left|f\left(t, t^{\beta-2} t^{2-\beta} y(t), t^{\beta-3} t^{3-\beta} y^{\prime}(t), t^{\beta-4} t^{4-\beta} y^{\prime \prime}(t), t^{\beta-n-2} t^{2+n-\beta} D_{0^{+}}^{n} y(t)\right)\right| \\
\leq & M_{r} t^{k_{1}}(1-t)^{\sigma_{1}} \quad \text { for all } t \in(0,1) .
\end{aligned}
$$

On the other hand, by direct computation, we can get the formulas for $\left(T_{1} y\right)^{\prime}(t),\left(T_{2} x\right)^{\prime \prime}(t), D_{0^{+}}^{m}\left(T_{1} y\right)(t)$. Similarly to Lemmas 2.1, from (11), we can prove that $T_{1} y \in X$. So $T_{1}: Y \rightarrow X$ is well defined.

SteP 2. $T_{1}$ is continuous.

Let $\left\{y_{u} \in Y\right\}$ be a sequence such that $y_{u} \rightarrow y_{0}$ in $Y$ as $u \rightarrow \infty$. Then there exists $r>0$ such that

$$
\begin{aligned}
& \max \left\{\sup _{t \in(0,1]} t^{2-\beta}\left|y_{u}(t)\right|, \sup _{t \in(0,1]} t^{3-\beta}\left|y_{u}^{\prime}(t)\right|,\right. \\
&\left.\sup _{t \in(0,1]} t^{4-\beta}\left|y_{u}^{\prime \prime}(t)\right|, \sup _{t \in(0,1]} t^{2+n-\beta}\left|D_{0^{+}}^{n} y_{u}(t)\right|\right\} \leq r
\end{aligned}
$$

for $n=0,1,2, \ldots$ Then there exist $M_{r}>0, k_{1}>-1$ and $\sigma_{1} \leq 0$ such that $2+\sigma_{1}+k_{1}>0$ and

$$
\left|f\left(t, y_{u}(t), y_{u}^{\prime}(t), y_{u}^{\prime \prime}(t), D_{0^{+}}^{n} y_{u}(t)\right)\right| \leq M_{r} t^{k_{1}}(1-t)^{\sigma_{1}}
$$

for all $t \in(0,1), n=0,1,2, \ldots$.

Denote $f_{y}(t)=f\left(t, y(t), y^{\prime}(t), y^{\prime \prime}(t), D_{0^{+}}^{n} y(t)\right)$. We have

$$
\begin{aligned}
t^{2-\alpha}\left|\left(T_{1} y_{u}\right)(t)-\left(T_{1} y_{0}\right)(t)\right| \leq & t^{2-\alpha} \int_{0}^{t} \frac{(t-s)^{\alpha-1}}{\Gamma(\alpha)} \mid f_{y_{u}}(s)-f_{y_{0}}(s \mid d s \\
& +\left|\frac{t}{\Gamma(\alpha)}-\frac{1}{\Gamma(\alpha-1)}\right| \int_{0}^{1} \mid f_{y_{u}}(s)-f_{y_{0}}(s \mid d s \\
& +\frac{1}{\Gamma(\alpha-1)} \int_{0}^{1}(1-s) \mid f_{y_{u}}(s)-f_{y_{0}}(s \mid d s
\end{aligned}
$$




$$
\begin{aligned}
& \leq t^{2-\alpha} \int_{0}^{t} \frac{(t-s)^{\alpha-1}}{\Gamma(\alpha)} 2 M_{r} s^{k_{1}}(1-s)^{\sigma_{1}} d s \\
& +\left[\frac{1}{\Gamma(\alpha)}+\frac{1}{\Gamma(\alpha-1)}\right] \int_{0}^{1} 2 M_{r} s^{k_{1}}(1-s)^{\sigma_{1}} d s \\
& +\frac{1}{\Gamma(\alpha-1)} \int_{0}^{1}(1-s) 2 M_{r} s^{k_{1}}(1-s)^{\sigma_{1}} d s \\
& \leq 2 M_{r} t^{2-\alpha} \int_{0}^{t} \frac{(t-s)^{\alpha-1}}{\Gamma(\alpha)} s^{k_{1}}(t-s)^{\sigma_{1}} d s \\
& +2 M_{r}\left[\frac{1}{\Gamma(\alpha)}+\frac{1}{\Gamma(\alpha-1)}\right] \int_{0}^{1} s^{k_{1}}(1-s)^{\sigma_{1}} d s \\
& +\frac{2 M_{r}}{\Gamma(\alpha-1)} \int_{0}^{1}(1-s) s^{k_{1}}(1-s)^{\sigma_{1}} d s \\
& =2 M_{r} t^{2+\sigma_{1}+k_{1}} \int_{0}^{1} \frac{(1-w)^{\alpha+\sigma_{1}-1}}{\Gamma(\alpha)} s^{k_{1}} d s \\
& +2 M_{r}\left[\frac{1}{\Gamma(\alpha)}+\frac{1}{\Gamma(\alpha-1)}\right] \mathbf{B}\left(\sigma_{1}+1, K_{1}+1\right) \\
& +\frac{2 M_{r}}{\Gamma(\alpha-1)} \mathbf{B}\left(2+\sigma_{1}, k_{1}+1\right) \\
& \leq 2 M_{r} \frac{\mathbf{B}\left(\alpha+\sigma_{1}, k_{1}+1\right)}{\Gamma(\alpha)} \\
& +2 M_{r}\left[\frac{1}{\Gamma(\alpha)}+\frac{1}{\Gamma(\alpha-1)}\right] \mathbf{B}\left(\sigma_{1}+1, K_{1}+1\right) \\
& +\frac{2 M_{r}}{\Gamma(\alpha-1)} \mathbf{B}\left(2+\sigma_{1}, k_{1}+1\right)
\end{aligned}
$$

and similarly

$$
\begin{aligned}
& t^{3-\alpha}\left|\left(T_{1} y_{u}\right)^{\prime}(t)-\left(T_{1} y_{0}\right)^{\prime}(t)\right| \leq 2 M_{r} \frac{\mathbf{B}\left(\alpha+\sigma_{1}-1, k_{1}+1\right)}{\Gamma(\alpha-1)} \\
& +2 M_{r}\left[\frac{1}{\Gamma(\alpha-1)}+\frac{1}{\Gamma(\alpha-2)}\right] \mathbf{B}\left(\sigma_{1}+1, K_{1}+1\right)+\frac{2 M_{r}}{\Gamma(\alpha-2)} \mathbf{B}\left(2+\sigma_{1}, k_{1}+1\right), \\
& t^{4-\alpha}\left|\left(T_{1} y_{u}\right)^{\prime \prime}(t)-\left(T_{1} y_{0}\right)^{\prime \prime}(t)\right| \leq 2 M_{r} \frac{\mathbf{B}\left(\alpha+\sigma_{1}-2, k_{1}+1\right)}{\Gamma(\alpha-2)} \\
& +2 M_{r}\left[\frac{1}{\Gamma(\alpha-2)}+\frac{1}{\Gamma(\alpha-3)}\right] \mathbf{B}\left(\sigma_{1}+1, K_{1}+1\right)+\frac{2 M_{r}}{\Gamma(\alpha-3)} \mathbf{B}\left(2+\sigma_{1}, k_{1}+1\right),
\end{aligned}
$$




$$
\begin{aligned}
t^{2+m-\alpha} \mid D_{0^{+}}^{m}\left(T_{1} y_{u}\right)(t) & -D_{0^{+}}^{m}\left(T_{1} y_{0}\right)(t) \mid \leq \\
+2 M_{r}\left[\frac{1}{\Gamma(\alpha-m)}+\right. & \frac{\mathbf{B}\left(\alpha+\sigma_{1}-m, k_{1}+1\right)}{\Gamma(\alpha-m)} \\
& \left.+\frac{1}{\Gamma(\alpha-m-1)}\right] \mathbf{B}\left(\sigma_{1}+1, K_{1}+1\right) \\
& +\frac{2 M_{r}}{\Gamma-1)} \mathbf{B}\left(2+\sigma_{1}, k_{1}+1\right) .
\end{aligned}
$$

By the dominant convergence theorem, we have

$$
\begin{aligned}
\lim _{n \rightarrow \infty} \sup _{t \in(0,1]} t^{2-\alpha} \mid\left(T_{1} y_{u}\right)(t) & -\left(T_{1} y_{0}\right)(t) \mid \\
= & \lim _{n \rightarrow \infty} \sup _{t \in(0,1]} t^{3-\alpha}\left|\left(T_{1} y_{u}\right)^{\prime}(t)-\left(T_{1} y_{0}\right)^{\prime}(t)\right|=0
\end{aligned}
$$

and

$$
\begin{aligned}
\lim _{n \rightarrow \infty} \sup _{t \in(0,1]} t^{4-\alpha}\left|\left(T_{1} y_{u}\right)^{\prime \prime}(t)-\left(T_{1} y_{0}\right)^{\prime \prime}(t)\right| & \\
& =\lim _{n \rightarrow \infty} \sup _{t \in(0,1]} t^{2+m-\alpha}\left|D_{0^{+}}^{m}\left(T_{1} y_{u}\right)(t)-D_{0^{+}}^{m}\left(T_{1} y_{0}\right)(t)\right|=0 .
\end{aligned}
$$

Thus $\left\|T_{1} y_{u}-T_{1} y_{0}\right\| \rightarrow 0$ as $u \rightarrow \infty$, so $T_{1}$ is continuous.

Now we prove that $T_{1}$ maps bounded sets in $Y$ into relatively compact sets in $X$. Let $\Omega \subset Y$ be a bounded subset. Then there exists $r>0$ such that $\|y\|_{Y} \leq r$ for all $y \in \Omega$.

Hence there exist $M_{r}>0, k_{1}>-1$ and $\sigma_{1} \leq 0$ such that $2+\sigma_{1}+k_{1}>0$ and (11) holds.

STEP 3. $\left\{T_{1} y: y \in \Omega\right\}$ is a bounded set in $X$ for every bounded $\Omega \subset Y$.

Similarly to Step 2, we can show that

$$
\begin{aligned}
& t^{2-\alpha}\left|\left(T_{1} y\right)(t)\right| \leq M_{r} \frac{\mathbf{B}\left(\alpha+\sigma_{1}, k_{1}+1\right)}{\Gamma(\alpha)} \\
& +M_{r}\left[\frac{1}{\Gamma(\alpha)}+\frac{1}{\Gamma(\alpha-1)}\right] \mathbf{B}\left(\sigma_{1}+1, K_{1}+1\right)+\frac{M_{r}}{\Gamma(\alpha-1)} \mathbf{B}\left(2+\sigma_{1}, k_{1}+1\right), \\
& t^{3-\alpha}\left|\left(T_{1} y\right)^{\prime}(t)\right| \leq M_{r} \frac{\mathbf{B}\left(\alpha+\sigma_{1}-1, k_{1}+1\right)}{\Gamma(\alpha-1)} \\
& +M_{r}\left[\frac{1}{\Gamma(\alpha-1)}+\frac{1}{\Gamma(\alpha-2)}\right] \mathbf{B}\left(\sigma_{1}+1, k_{1}+1\right)+\frac{M_{r}}{\Gamma(\alpha-2)} \mathbf{B}\left(2+\sigma_{1}, k_{1}+1\right), \\
& t^{4-\alpha}\left|\left(T_{1} y\right)^{\prime \prime}(t)\right| \leq M_{r} \frac{\mathbf{B}\left(\alpha+\sigma_{1}-2, k_{1}+1\right)}{\Gamma(\alpha-2)} \\
& +M_{r}\left[\frac{1}{\Gamma(\alpha-2)}+\frac{1}{\Gamma(\alpha-3)}\right] \mathbf{B}\left(\sigma_{1}+1, K_{1}+1\right)+\frac{M_{r}}{\Gamma(\alpha-3)} \mathbf{B}\left(2+\sigma_{1}, k_{1}+1\right),
\end{aligned}
$$




$$
\begin{aligned}
t^{2+m-\alpha}\left|D_{0^{+}}^{m}\left(T_{1} y\right)(t)\right| \leq & M_{r} \frac{\mathbf{B}\left(\alpha+\sigma_{1}-m, k_{1}+1\right)}{\Gamma(\alpha-m)} \\
& +M_{r}\left[\frac{1}{\Gamma(\alpha-m)}+\frac{1}{\Gamma(\alpha-m-1)}\right] \mathbf{B}\left(\sigma_{1}+1, K_{1}+1\right) \\
& +\frac{M_{r}}{\Gamma(\alpha-m-1)} \mathbf{B}\left(2+\sigma_{1}, k_{1}+1\right) .
\end{aligned}
$$

So $T_{1}$ maps bounded sets into bounded sets in $X$.

STEP 4. $\left\{T_{1} y: y \in \Omega\right\}$ is a relatively compact set in $X$ for every bounded set $\Omega \subset Y$.

We prove that

$$
\begin{array}{ll}
\left\{t^{2-\alpha}\left(T_{1} y\right)(t): y \in \Omega\right\}, & \left\{t^{3-\alpha}\left(T_{1} y\right)^{\prime}(t): y \in \Omega\right\}, \\
\left\{t^{4-\alpha}\left(T_{1} y\right)^{\prime \prime}(t): y \in \Omega\right\}, & \left\{t^{2+m-\alpha} D_{0^{+}}^{m}\left(T_{1} y\right)(t): y \in \Omega\right\}
\end{array}
$$

are equi-continuous on $(0,1]$.

First, let $t_{1}, t_{2} \in[e, d] \subset(0,1], t_{1}<t_{2}, 0<e<d \leq 1$, and $y \in \Omega$. Then

$$
\begin{aligned}
\left|t_{1}^{2-\alpha}\left(T_{1} y\right)\left(t_{1}\right)-t_{2}^{2-\alpha}\left(T_{1} y\right)\left(t_{2}\right)\right| & \mid t_{1}^{2-\alpha} \int_{0}^{t_{1}} \frac{\left(t_{1}-s\right)^{\alpha-1}}{\Gamma(\alpha)} f\left(s, y(s), y^{\prime}(s), y^{\prime \prime}(s), D_{0^{+}}^{n} y(s)\right) d s \\
& -t_{2}^{2-\alpha} \int_{0}^{t_{2}} \frac{\left(t_{2}-s\right)^{\alpha-1}}{\Gamma(\alpha)} f\left(s, y(s), y^{\prime}(s), y^{\prime \prime}(s), D_{0^{+}}^{n} y(s)\right) d s \mid \\
& +\frac{1}{\Gamma(\alpha)}\left|t_{1}-t_{2}\right| \int_{0}^{1}\left|f\left(s, y(s), y^{\prime}(s), y^{\prime \prime}(s), D_{0^{+}}^{n} y(s)\right)\right| d s \\
\leq & \mid t_{1}^{2-\alpha} \int_{0}^{t_{1}} \frac{\left(t_{1}-s\right)^{\alpha-1}}{\Gamma(\alpha)} f\left(s, y(s), y^{\prime}(s), y^{\prime \prime}(s), D_{0^{+}}^{n} y(s)\right) d s \\
& \quad-t_{2}^{2-\alpha} \int_{0}^{t_{2}} \frac{\left(t_{2}-s\right)^{\alpha-1}}{\Gamma(\alpha)} f\left(s, y(s), y^{\prime}(s), y^{\prime \prime}(s), D_{0^{+}}^{n} y(s)\right) d s \mid \\
& +\frac{M_{r}}{\Gamma(\alpha)}\left|t_{1}-t_{2}\right| \int_{0}^{1} s^{k_{1}}(1-s)^{\sigma_{1}} d s .
\end{aligned}
$$

We find that

$$
\begin{aligned}
\mid t_{1}^{2-\alpha} \int_{0}^{t_{1}} \frac{\left(t_{1}-s\right)^{\alpha-1}}{\Gamma(\alpha)} f & \left(s, y(s), y^{\prime}(s), y^{\prime \prime}(s), D_{0^{+}}^{n} y(s)\right) d s \\
& -t_{2}^{2-\alpha} \int_{0}^{t_{2}} \frac{\left(t_{2}-s\right)^{\alpha-1}}{\Gamma(\alpha)} f\left(s, y(s), y^{\prime}(s), y^{\prime \prime}(s), D_{0^{+}}^{n} y(s)\right) d s \mid
\end{aligned}
$$




$$
\begin{aligned}
& \leq\left|t_{2}^{2-\alpha}-t_{1}^{2-\alpha}\right| \int_{0}^{t_{1}} \frac{\left(t_{1}-s\right)^{\alpha-1}}{\Gamma(\alpha)}\left|f\left(s, y(s), y^{\prime}(s), y^{\prime \prime}(s), D_{0^{+}}^{n} y(s)\right)\right| d s \\
& +t_{2}^{2-\alpha} \int_{t_{1}}^{t_{2}} \frac{\left(t_{2}-s\right)^{\alpha-1}}{\Gamma(\alpha)}\left|f\left(s, y(s), y^{\prime}(s), y^{\prime \prime}(s), D_{0^{+}}^{n} y(s)\right)\right| d s \\
& +t_{2}^{2-\alpha} \int_{0}^{t_{2}} \frac{\left|\left(t_{1}-s\right)^{\alpha-1}-\left(t_{2}-s\right)^{\alpha-1}\right|}{\Gamma(\alpha)}\left|f\left(s, y(s), y^{\prime}(s), y^{\prime \prime}(s), D_{0^{+}}^{n} y(s)\right)\right| d s \\
& \leq\left|t_{2}^{2-\alpha}-t_{1}^{2-\alpha}\right| \int_{0}^{t_{1}} \frac{\left(t_{1}-s\right)^{\alpha-1}}{\Gamma(\alpha)} M_{r} s^{k_{1}}(1-s)^{\sigma_{1}} d s \\
& +t_{2}^{2-\alpha} \int_{t_{1}}^{t_{2}} \frac{\left(t_{2}-s\right)^{\alpha-1}}{\Gamma(\alpha)} M_{r} s^{k_{1}}(1-s)^{\sigma_{1}} d s \\
& +t_{2}^{2-\alpha} \int_{0}^{t_{2}} \frac{\left|\left(t_{1}-s\right)^{\alpha-1}-\left(t_{2}-s\right)^{\alpha-1}\right|}{\Gamma(\alpha)} M_{r} s^{k_{1}}(1-s)^{\sigma_{1}} d s \\
& \leq M_{r}\left[e^{4-2 \alpha}\left|t_{2}^{\alpha-2}-t_{1}^{\alpha-2}\right| \int_{0}^{t_{1}} \frac{\left(t_{1}-s\right)^{\alpha-1}}{\Gamma(\alpha)} s^{k_{1}}\left(t_{1}-s\right)^{\sigma_{1}} d s\right. \\
& +e^{2-\alpha} \int_{t_{1}}^{t_{2}} \frac{\left(t_{2}-s\right)^{\alpha-1}}{\Gamma(\alpha)} s^{k_{1}}\left(t_{2}-s\right)^{\sigma_{1}} d s \\
& \left.+t_{2}^{2-\alpha} \int_{0}^{t_{2}} \frac{\left(t_{2}-s\right)^{\alpha-1}-\left(t_{1}-s\right)^{\alpha-1}}{\Gamma(\alpha)} s^{k_{1}}(1-s)^{\sigma_{1}} d s\right] \\
& =M_{r}\left[e^{4-2 \alpha}\left|t_{2}^{\alpha-2}-t_{1}^{\alpha-2}\right| t_{1}^{\alpha+k_{1}+\sigma_{1}} \int_{0}^{1} \frac{(1-w)^{\alpha+\sigma_{1}-1}}{\Gamma(\alpha)} w^{k_{1}} d w\right. \\
& +e^{2-\alpha} t_{2}^{\alpha+k_{1}+\sigma_{1}} \int_{t_{1} / t_{2}}^{1} \frac{(1-w)^{\alpha+\sigma_{1}-1}}{\Gamma(\alpha)} w^{k_{1}} d w \\
& \left.+e^{2-\alpha} \int_{0}^{t_{2}} \frac{\left(t_{2}-s\right)^{\alpha-1}-\left(t_{1}-s\right)^{\alpha-1}}{\Gamma(\alpha)} s^{k_{1}}(1-s)^{\sigma_{1}} d s\right] .
\end{aligned}
$$

We can prove that $1-x^{\tau} \leq \frac{\tau}{\tau-2}(1-x)^{\tau-2}$ for all $x \in[0,1]$ and $\tau \in(2,3)$. In fact, let $g(x)=1-x^{\tau}-\frac{\tau}{\tau-2}(1-x)^{\tau-2}$. It is easy to see that $g(1)=0$ and

$$
g^{\prime}(x)=-\tau x^{\tau-1}+\tau(1-x)^{\tau-3} \geq 0, \quad x \in(0,1) .
$$

Thus $g(x) \leq g(1)=0$ for all $x \in[0,1]$. Choose $\tau=\alpha-1$ and $x=v / u$. It follows that

$$
u^{\alpha-2}-\frac{v^{\alpha-1}}{u} \leq \frac{\alpha-1}{\alpha-3} u(u-v)^{\alpha-3}, \quad u \geq v .
$$


Hence

$$
\begin{aligned}
& \mid t_{1}^{2-\alpha} \int_{0}^{t_{1}} \frac{\left(t_{1}-s\right)^{\alpha-1}}{\Gamma(\alpha)} f\left(s, y(s), y^{\prime}(s), y^{\prime \prime}(s), D_{0^{+}}^{n} y(s)\right) d s \\
& -t_{2}^{2-\alpha} \int_{0}^{t_{2}} \frac{\left(t_{2}-s\right)^{\alpha-1}}{\Gamma(\alpha)} f\left(s, y(s), y^{\prime}(s), y^{\prime \prime}(s), D_{0^{+}}^{n} y(s)\right) d s \mid \\
& \leq M_{r}\left[e^{4-2 \alpha}\left|t_{2}^{\alpha-2}-t_{1}^{\alpha-2}\right| \frac{\mathbf{B}\left(\alpha+\sigma_{1}, k_{1}+1\right)}{\Gamma(\alpha)}\right. \\
& +e^{2-\alpha} \int_{t_{1} / t_{2}}^{1} \frac{(1-w)^{\alpha+\sigma_{1}-1}}{\Gamma(\alpha)} w^{k_{1}} d w \\
& \left.+e^{2-\alpha} \int_{0}^{1} \frac{\frac{\alpha-1}{\alpha-3}\left(t_{2}-t_{1}\right)^{\alpha-1}}{\Gamma(\alpha)} s^{k_{1}}(1-s)^{\sigma_{1}} d s\right] \\
& \leq M_{r}\left[e^{4-2 \alpha}\left|t_{2}^{\alpha-2}-t_{1}^{\alpha-2}\right| \frac{\mathbf{B}\left(\alpha+\sigma_{1}, k_{1}+1\right)}{\Gamma(\alpha)}\right. \\
& +e^{2-\alpha} \int_{t_{1} / t_{2}}^{1} \frac{(1-w)^{\alpha+\sigma_{1}-1}}{\Gamma(\alpha)} w^{k_{1}} d w \\
& \left.+e^{2-\alpha} \frac{\alpha-1}{\alpha-3}\left(t_{2}-t_{1}\right)^{\alpha-1} \frac{1}{\Gamma(\alpha)} \mathbf{B}\left(\sigma_{1}+1, k_{1}+1\right)\right] .
\end{aligned}
$$

As $t_{1} \rightarrow t_{2}$, the right-hand side tends to zero uniformly. So

$$
\begin{aligned}
&\left|t_{1}^{2-\alpha}\left(T_{1} y\right)\left(t_{1}\right)-t_{2}^{2-\alpha}\left(T_{1} y\right)\left(t_{2}\right)\right| \rightarrow 0 \\
& \text { uniformly as } t_{1} \rightarrow t_{2} \text { on }[e, d] \subseteq(0,1] .
\end{aligned}
$$

Furthermore,

$$
\begin{aligned}
\mid t^{2-\alpha}\left(T_{1} y\right)(t)- & \left(-\frac{1}{\Gamma(\alpha-1)} \int_{0}^{1} f\left(s, y(s), y^{\prime}(s), y^{\prime \prime}(s), D_{0^{+}}^{n} y(s)\right) d\right. \\
& \left.+\frac{1}{\Gamma(\alpha-1)} \int_{0}^{1}(1-s) f\left(s, y(s), y^{\prime}(s), y^{\prime \prime}(s), D_{0^{+}}^{n} y(s)\right) d s\right) \mid \\
\leq & t^{2-\alpha} \int_{0}^{t} \frac{(t-s)^{\alpha-1}}{\Gamma(\alpha)}\left|f\left(s, y(s), y^{\prime}(s), y^{\prime \prime}(s), D_{0^{+}}^{n} y(s)\right)\right| d s \\
& +\frac{1}{\Gamma(\alpha)} t \int_{0}^{1}\left|f\left(s, y(s), y^{\prime}(s), y^{\prime \prime}(s), D_{0^{+}}^{n} y(s)\right)\right| d s \\
\leq & M_{r} t^{2-\alpha} \int_{0}^{t} \frac{(t-s)^{\alpha-1}}{\Gamma(\alpha)} s^{k_{1}}(1-s)^{\sigma_{1}} d s+\frac{M_{r}}{\Gamma(\alpha)} t \int_{0}^{1} s^{k_{1}}(1-s)^{\sigma_{1}} d s \\
\leq & M_{r} t^{2+\sigma_{1}+k_{1}} \int_{0}^{1} \frac{(1-w)^{\alpha+\sigma_{1}-1}}{\Gamma(\alpha)} w^{k_{1}} d w+\frac{M_{r}}{\Gamma(\alpha)} t \mathbf{B}\left(\sigma_{1}, k_{1}+1\right) .
\end{aligned}
$$


It follows that

$$
t^{2-\alpha}\left(T_{1} y\right)(t) \text { is uniformly convergent as } t \rightarrow 0 \text {. }
$$

From (12) and (13), we know that $\left\{t^{2-\beta}\left(T_{1} y\right)(t): y \in \Omega\right\}$ is equi-continuous on $(0,1]$.

Secondly, let $t_{1}, t_{2} \in[e, d] \subset(0,1]$ with $t_{1}<t_{2}, 0<e<d \leq 1$, and $y \in \Omega$. Then

$$
\begin{aligned}
\mid t_{1}^{3-\alpha}\left(T_{1} y\right)^{\prime}\left(t_{1}\right)- & t_{2}^{3-\alpha}\left(T_{1} y\right)^{\prime}\left(t_{2}\right) \mid \\
\leq & \mid t_{1}^{3-\alpha} \int_{0}^{t_{1}} \frac{\left(t_{1}-s\right)^{\alpha-2}}{\Gamma(\alpha-1)} f\left(s, y(s), y^{\prime}(s), y^{\prime \prime}(s), D_{0^{+}}^{n} y(s)\right) d s \\
& -t_{2}^{3-\alpha} \int_{0}^{t_{2}} \frac{\left(t_{2}-s\right)^{\alpha-2}}{\Gamma(\alpha-1)} f\left(s, y(s), y^{\prime}(s), y^{\prime \prime}(s), D_{0^{+}}^{n} y(s)\right) d s \mid \\
& +\left|t_{1}-t_{2}\right| \frac{1}{\Gamma(\alpha-1)} \int_{0}^{1}\left|f\left(s, y(s), y^{\prime}(s), y^{\prime \prime}(s), D_{0^{+}}^{n} y(s)\right)\right| d s \\
\leq & \mid t_{1}^{3-\alpha} \int_{0}^{t_{1}} \frac{\left(t_{1}-s\right)^{\alpha-2}}{\Gamma(\alpha-1)} f\left(s, y(s), y^{\prime}(s), y^{\prime \prime}(s), D_{0^{+}}^{n} y(s)\right) d s \\
& \quad-t_{2}^{3-\alpha} \int_{0}^{t_{2}} \frac{\left(t_{2}-s\right)^{\alpha-2}}{\Gamma(\alpha-1)} f\left(s, y(s), y^{\prime}(s), y^{\prime \prime}(s), D_{0^{+}}^{n} y(s)\right) d s \mid \\
& +M_{r}\left|t_{1}-t_{2}\right| \frac{1}{\Gamma(\alpha-1)} \mathbf{B}\left(\sigma_{1}+1, k_{1}+1\right) .
\end{aligned}
$$

We find that

$$
\begin{aligned}
& \mid t_{1}^{3-\alpha} \int_{0}^{t_{1}} \frac{\left(t_{1}-s\right)^{\alpha-2}}{\Gamma(\alpha-1)} f\left(s, y(s), y^{\prime}(s), y^{\prime \prime}(s), D_{0^{+}}^{n} y(s)\right) d s \\
& \quad-t_{2}^{3-\alpha} \int_{0}^{t_{2}} \frac{\left(t_{2}-s\right)^{\alpha-2}}{\Gamma(\alpha-1)} f\left(s, y(s), y^{\prime}(s), y^{\prime \prime}(s), D_{0^{+}}^{n} y(s)\right) d s \mid \\
& \leq\left|t_{2}^{3-\alpha}-t_{1}^{3-\alpha}\right| \int_{0}^{t_{1}} \frac{\left(t_{1}-s\right)^{\alpha-2}}{\Gamma(\alpha-1)}\left|f\left(s, y(s), y^{\prime}(s), y^{\prime \prime}(s), D_{0^{+}}^{n} y(s)\right)\right| d s \\
& \quad+t_{2}^{3-\alpha} \int_{t_{1}}^{t_{2}} \frac{\left(t_{1}-s\right)^{\alpha-2}}{\Gamma(\alpha-1)}\left|f\left(s, y(s), y^{\prime}(s), y^{\prime \prime}(s), D_{0^{+}}^{n} y(s)\right)\right| d s \\
& \quad+t_{2}^{3-\alpha} \int_{0}^{t_{2}} \frac{\left|\left(t_{1}-s\right)^{\alpha-2}-\left(t_{2}-s\right)^{\alpha-2}\right|}{\Gamma(\alpha-1)}\left|f\left(s, y(s), y^{\prime}(s), y^{\prime \prime}(s), D_{0^{+}}^{n} y(s)\right)\right| d s
\end{aligned}
$$




$$
\begin{aligned}
\leq & M_{r}\left[e^{6-2 \alpha}\left|t_{2}^{\alpha-3}-t_{1}^{\alpha-3}\right| t_{1}^{\alpha+k_{1}+\sigma_{1}-1} \int_{0}^{1} \frac{(1-w)^{\alpha+\sigma_{1}-2}}{\Gamma(\alpha-1)} w^{k_{1}} d w\right. \\
& +e^{3-\alpha} t_{2}^{\alpha+k_{1}+\sigma_{1}-1} \int_{t_{1} / t_{2}}^{1} \frac{(1-w)^{\alpha+\sigma_{1}-2}}{\Gamma(\alpha-1)} w^{k_{1}} d w \\
& \left.+e^{3-\alpha} \int_{0}^{t_{2}} \frac{\left(t_{2}-s\right)^{\alpha-2}-\left(t_{1}-s\right)^{\alpha-2}}{\Gamma(\alpha-1)} s^{k_{1}}(1-s)^{\sigma_{1}} d s\right]
\end{aligned}
$$

We can prove that $1-x^{\tau} \leq \frac{\tau}{\tau-1}(1-x)^{\tau-1}$ for all $x \in[0,1]$ and $\tau \in(1,2)$. In fact, let $g(x)=1-x^{\tau}-\frac{\tau}{\tau-1}(1-x)^{\tau-1}$. It is easy to see that $g(1)=0$ and

$$
g^{\prime}(x)=-\tau x^{\tau-1}+\tau(1-x)^{\tau-2} \geq 0, \quad x \in(0,1) .
$$

Thus $g(x) \leq g(1)=0$ for all $x \in[0,1]$. Choose $\tau=\alpha-2$ and $x=v / u$. Then

\section{Hence}

$$
u^{\alpha-2}-v^{\alpha-2} \leq \frac{\alpha-2}{\alpha-3}(u-v)^{\alpha-3}, \quad u \geq v .
$$

$$
\begin{aligned}
\mid t_{1}^{3-\alpha} \int_{0}^{t_{1}} \frac{\left(t_{1}-s\right)^{\alpha-2}}{\Gamma(\alpha-1)} f & \left.s, y(s), y^{\prime}(s), y^{\prime \prime}(s), D_{0^{+}}^{n} y(s)\right) d s \\
& -t_{2}^{3-\alpha} \int_{0}^{t_{2}} \frac{\left(t_{2}-s\right)^{\alpha-2}}{\Gamma(\alpha-1)} f\left(s, y(s), y^{\prime}(s), y^{\prime \prime}(s), D_{0^{+}}^{n} y(s)\right) d s \mid \\
\leq & M_{r}\left[e^{6-2 \alpha}\left|t_{2}^{\alpha-3}-t_{1}^{\alpha-3}\right| \frac{\mathbf{B}\left(\alpha+\sigma_{1}-1, k_{1}+1\right)}{\Gamma(\alpha-1)}\right. \\
& +e^{3-\alpha} \int_{t_{1} / t_{2}}^{1} \frac{(1-w)^{\alpha+\sigma_{1}-2}}{\Gamma(\alpha-1)} w^{k_{1}} d w \\
& \left.+e^{3-\alpha} \frac{\alpha-2}{\alpha-3}\left(t_{2}-t_{1}\right)^{\alpha-3} \frac{1}{\Gamma(\alpha-1)} \int_{0}^{1} s^{k_{1}}(1-s)^{\sigma_{1}} d s\right] .
\end{aligned}
$$

As $t_{1} \rightarrow t_{2}$, the right-hand side tends to zero uniformly. So

$$
\begin{aligned}
&\left|t_{1}^{3-\alpha}\left(T_{1} y\right)^{\prime}\left(t_{1}\right)-t_{2}^{3-\alpha}\left(T_{1} y\right)^{\prime}\left(t_{2}\right)\right| \rightarrow 0 \\
& \text { uniformly as } t_{1} \rightarrow t_{2} \text { on }[e, d] \subseteq(0,1] .
\end{aligned}
$$

Furthermore,

$$
\begin{aligned}
\mid t^{3-\alpha}\left(T_{1} y\right)^{\prime}(t)-( & -\frac{1}{\Gamma(\alpha-2)} \int_{0}^{1} f\left(s, y(s), y^{\prime}(s), y^{\prime \prime}(s), D_{0^{+}}^{n} y(s)\right) d s \\
& \left.+\frac{1}{\Gamma(\alpha-2)} \int_{0}^{1}(1-s) f\left(s, y(s), y^{\prime}(s), y^{\prime \prime}(s), D_{0^{+}}^{n} y(s)\right) d s\right) \mid
\end{aligned}
$$




$$
\begin{aligned}
& \leq t^{3-\alpha} \int_{0}^{t} \frac{(t-s)^{\alpha-2}}{\Gamma(\alpha-1)}\left|f\left(s, y(s), y^{\prime}(s), y^{\prime \prime}(s), D_{0^{+}}^{n} y(s)\right)\right| d s \\
& \quad+\frac{1}{\Gamma(\alpha-1)} t \int_{0}^{1}\left|f\left(s, y(s), y^{\prime}(s), y^{\prime \prime}(s), D_{0^{+}}^{n} y(s)\right)\right| d s \\
& \leq M_{r} t^{3-\alpha} \int_{0}^{t} \frac{(t-s)^{\alpha-2}}{\Gamma(\alpha-1)} s^{k_{1}}(1-s)^{\sigma_{1}} d s+\frac{M_{r}}{\Gamma(\alpha-1)} t \int_{0}^{1} s^{k_{1}}(1-s)^{\sigma_{1}} d s \\
& \leq M_{r} t^{2+\sigma_{1}+k_{1}} \int_{0}^{1} \frac{(1-w)^{\alpha+\sigma_{1}-2}}{\Gamma(\alpha-1)} w^{k_{1}} d w+\frac{M_{r}}{\Gamma(\alpha-1)} t \mathbf{B}\left(\sigma_{1}, k_{1}+1\right) .
\end{aligned}
$$

It follows that

$$
t^{3-\alpha}\left(T_{1} y\right)^{\prime}(t) \text { is uniformly convergent as } t \rightarrow 0 \text {. }
$$

From (14) and (15), we know that $\left\{t^{3-\alpha}\left(T_{1} y\right)^{\prime}(t): y \in \Omega\right\}$ is equi-continuous on $(0,1]$.

Thirdly, let $t_{1}, t_{2} \in(0,1]$ with $t_{1}<t_{2}$ and $y \in \Omega$. Then

$$
\begin{aligned}
\mid t_{1}^{4-\alpha}\left(T_{1} y\right)^{\prime \prime}\left(t_{1}\right)- & t_{2}^{4-\alpha}\left(T_{1} y\right)^{\prime \prime}\left(t_{2}\right) \mid \\
\leq & \mid t_{1}^{4-\alpha} \int_{0}^{t_{1}} \frac{\left(t_{1}-s\right)^{\alpha-3}}{\Gamma(\alpha-2)} f\left(s, y(s), y^{\prime}(s), y^{\prime \prime}(s), D_{0^{+}}^{n} y(s)\right) d s \\
& \quad-t_{2}^{4-\alpha} \int_{0}^{t_{2}} \frac{\left(t_{2}-s\right)^{\alpha-3}}{\Gamma(\alpha-2)} f\left(s, y(s), y^{\prime}(s), y^{\prime \prime}(s), D_{0^{+}}^{n} y(s)\right) d s \mid \\
& +\left|t_{1}-t_{2}\right| \frac{1}{\Gamma(\alpha-2)} \int_{0}^{1}\left|f\left(s, y(s), y^{\prime}(s), y^{\prime \prime}(s), D_{0^{+}}^{n} y(s)\right)\right| d s \\
\leq & \mid t_{1}^{4-\alpha} \int_{0}^{t_{1}} \frac{\left(t_{1}-s\right)^{\alpha-3}}{\Gamma(\alpha-2)} f\left(s, y(s), y^{\prime}(s), y^{\prime \prime}(s), D_{0^{+}}^{n} y(s)\right) d s \\
& -t_{2}^{4-\alpha} \int_{0}^{t_{2}} \frac{\left(t_{2}-s\right)^{\alpha-3}}{\Gamma(\alpha-2)} f\left(s, y(s), y^{\prime}(s), y^{\prime \prime}(s), D_{0^{+}}^{n} y(s)\right) d s \mid \\
& +M_{r}\left|t_{1}-t_{2}\right| \frac{1}{\Gamma(\alpha-2)} \mathbf{B}\left(\sigma_{1}+1, k_{1}+1\right) .
\end{aligned}
$$

We find that

$$
\begin{aligned}
& \mid t_{1}^{4-\alpha} \int_{0}^{t_{1}} \frac{\left(t_{1}-s\right)^{\alpha-3}}{\Gamma(\alpha-2)} f\left(s, y(s), y^{\prime}(s), y^{\prime \prime}(s), D_{0^{+}}^{n} y(s)\right) d s \\
& \quad-t_{2}^{4-\alpha} \int_{0}^{t_{2}} \frac{\left(t_{2}-s\right)^{\alpha-3}}{\Gamma(\alpha-2)} f\left(s, y(s), y^{\prime}(s), y^{\prime \prime}(s), D_{0^{+}}^{n} y(s)\right) d s \mid
\end{aligned}
$$




$$
\begin{aligned}
& \leq\left|t_{2}^{4-\alpha}-t_{1}^{4-\alpha}\right| \int_{0}^{t_{1}} \frac{\left(t_{1}-s\right)^{\alpha-3}}{\Gamma(\alpha-2)}\left|f\left(s, y(s), y^{\prime}(s), y^{\prime \prime}(s), D_{0^{+}}^{n} y(s)\right)\right| d s \\
& +t_{2}^{4-\alpha} \int_{t_{1}}^{t_{2}} \frac{\left(t_{1}-s\right)^{\alpha-3}}{\Gamma(\alpha-2)}\left|f\left(s, y(s), y^{\prime}(s), y^{\prime \prime}(s), D_{0^{+}}^{n} y(s)\right)\right| d s \\
& +t_{2}^{4-\alpha} \int_{0}^{t_{2}} \frac{\left|\left(t_{1}-s\right)^{\alpha-3}-\left(t_{2}-s\right)^{\alpha-3}\right|}{\Gamma(\alpha-2)}\left|f\left(s, y(s), y^{\prime}(s), y^{\prime \prime}(s), D_{0^{+}}^{n} y(s)\right)\right| d s \\
& \leq M_{r}\left[\left|t_{2}^{4-\alpha}-t_{1}^{4-\alpha}\right| \int_{0}^{t_{1}} \frac{\left(t_{1}-s\right)^{\alpha-3}}{\Gamma(\alpha-2)} s^{k_{1}}(1-s)^{\sigma_{1}} d s\right. \\
& +t_{2}^{4-\alpha} \int_{t_{1}}^{t_{2}} \frac{\left(t_{1}-s\right)^{\alpha-3}}{\Gamma(\alpha-2)} s^{k_{1}}(1-s)^{\sigma_{1}} d s \\
& \left.+t_{2}^{4-\alpha} \int_{0}^{t_{2}} \frac{\left|\left(t_{1}-s\right)^{\alpha-3}-\left(t_{2}-s\right)^{\alpha-3}\right|}{\Gamma(\alpha-2)} s^{k_{1}}(1-s)^{\sigma_{1}} d s\right] \\
& \leq M_{r}\left[\left|t_{2}^{4-\alpha}-t_{1}^{4-\alpha}\right| \int_{0}^{t_{1}} \frac{\left(t_{1}-s\right)^{\alpha-3}}{\Gamma(\alpha-2)} s^{k_{1}}\left(t_{1}-s\right)^{\sigma_{1}} d s\right. \\
& +t_{2}^{4-\alpha} \int_{t_{1}}^{t_{2}} \frac{\left(t_{1}-s\right)^{\alpha-3}}{\Gamma(\alpha-2)} s^{k_{1}}\left(t_{2}-s\right)^{\sigma_{1}} d s \\
& \left.+t_{2}^{4-\alpha} \int_{0}^{t_{2}} \frac{\left|\left(t_{1}-s\right)^{\alpha-3}-\left(t_{2}-s\right)^{\alpha-3}\right|}{\Gamma(\alpha-2)} s^{k_{1}}(1-s)^{\sigma_{1}} d s\right] .
\end{aligned}
$$

We can prove that $1-x^{\tau} \leq(1-x)^{\tau}$ for all $x \in[0,1]$ and $\tau \in(0,1)$. Choose $\tau=\alpha-3$ and $x=v / u$. Then $u^{\alpha-3}-v^{\alpha-3} \leq(u-v)^{\alpha-3}$ if $u \geq v$. Hence

$$
\begin{aligned}
\mid t_{1}^{4-\alpha} \int_{0}^{t_{1}} \frac{\left(t_{1}-s\right)^{\alpha-3}}{\Gamma(\alpha-2)} f\left(s, y(s), y^{\prime}(s), y^{\prime \prime}(s), D_{0^{+}}^{n} y(s)\right) d s & \\
& -t_{2}^{4-\alpha} \int_{0}^{t_{2}} \frac{\left(t_{2}-s\right)^{\alpha-3}}{\Gamma(\alpha-2)} f\left(s, y(s), y^{\prime}(s), y^{\prime \prime}(s), D_{0^{+}}^{n} y(s)\right) d s \mid \\
\leq & M_{r}\left[\left|t_{2}^{4-\alpha}-t_{1}^{4-\alpha}\right| \frac{\mathbf{B}\left(\alpha+\sigma_{1}-2, k_{1}+1\right)}{\Gamma(\alpha-2)}\right. \\
& +\int_{1}^{1} \frac{(1-w)^{\alpha+\sigma_{1}-3}}{\Gamma(\alpha-2)} w^{k_{1}} d w \\
& \left.+t_{2}^{4-\alpha}\left(t_{2}-t_{1}\right)^{\alpha-3} \frac{1}{\Gamma(\alpha-2)} \mathbf{B}\left(\sigma_{1}+1, k_{1}+1\right)\right] .
\end{aligned}
$$


As $t_{1} \rightarrow t_{2}$, the right-hand side tends to zero uniformly. So

(16) $\left|t_{1}^{4-\alpha}\left(T_{1} y\right)^{\prime \prime}\left(t_{1}\right)-t_{2}^{4-\alpha}\left(T_{1} y\right)^{\prime \prime}\left(t_{2}\right)\right| \rightarrow 0$ uniformly as $t_{1} \rightarrow t_{2}$ on $(0,1]$.

Fourthly, let $t_{1}, t_{2} \in[e, d] \subset(0,1]$ with $0<e \leq t_{1}<t_{2} \leq d \leq 1$ and $y \in \Omega$. Then

$$
\begin{aligned}
\mid t_{1}^{2+m-\alpha} D_{0^{+}}^{m} & \left(T_{1} y\right)\left(t_{1}\right)-t_{2}^{2+m-\alpha} D_{0^{+}}^{m}\left(T_{1} y\right)\left(t_{2}\right) \mid \\
\leq & \mid t_{1}^{2+m-\alpha} \int_{0}^{t_{1}} \frac{\left(t_{1}-s\right)^{\alpha-m-1}}{\Gamma(\alpha-m)} f\left(s, y(s), y^{\prime}(s), y^{\prime \prime}(s), D_{0^{+}}^{n} y(s)\right) d s \\
& -t_{2}^{2+m-\alpha} \int_{0}^{t_{2}} \frac{\left(t_{2}-s\right)^{\alpha-m-1}}{\Gamma(\alpha-m)} f\left(s, y(s), y^{\prime}(s), y^{\prime \prime}(s), D_{0^{+}}^{n} y(s)\right) d s \mid \\
& +\left|t_{1}-t_{2}\right| \frac{1}{\Gamma(\alpha-m)} \int_{0}^{1}\left|f\left(s, y(s), y^{\prime}(s), y^{\prime \prime}(s), D_{0^{+}}^{n} y(s)\right)\right| d s \\
\leq & \mid t_{1}^{2+m-\alpha} \int_{0}^{t_{1}} \frac{\left(t_{1}-s\right)^{\alpha-m-1}}{\Gamma(\alpha-m)} f\left(s, y(s), y^{\prime}(s), y^{\prime \prime}(s), D_{0^{+}}^{n} y(s)\right) d s \\
& \quad-t_{2}^{2+m-\alpha} \int_{0}^{t_{2}} \frac{\left(t_{2}-s\right)^{\alpha-m-1}}{\Gamma(\alpha-m)} f\left(s, y(s), y^{\prime}(s), y^{\prime \prime}(s), D_{0^{+}}^{n} y(s)\right) d s \mid \\
& +M_{r}\left|t_{1}-t_{2}\right| \frac{1}{\Gamma(\alpha-m)} \mathbf{B}\left(\sigma_{1}+1, k_{1}+1\right) .
\end{aligned}
$$

We find that

$$
\begin{aligned}
& \mid t_{1}^{2+m-\alpha} \int_{0}^{t_{1}} \frac{\left(t_{1}-s\right)^{\alpha-m-1}}{\Gamma(\alpha-m)} f\left(s, y(s), y^{\prime}(s), y^{\prime \prime}(s), D_{0^{+}}^{n} y(s)\right) d s \\
& \quad-t_{2}^{2+m-\alpha} \int_{0}^{t_{2}} \frac{\left(t_{2}-s\right)^{\alpha-m-1}}{\Gamma(\alpha-m)} f\left(s, y(s), y^{\prime}(s), y^{\prime \prime}(s), D_{0^{+}}^{n} y(s)\right) d s \mid \\
& \leq\left|t_{2}^{2+m-\alpha}-t_{1}^{+m-\alpha}\right| \int_{0}^{t_{2}} \frac{\left(t_{2}-s\right)^{\alpha-m-1}}{\Gamma(\alpha-m)}\left|f\left(s, y(s), y^{\prime}(s), y^{\prime \prime}(s), D_{0^{+}}^{n} y(s)\right)\right| d s \\
& +t_{1}^{2+m-\alpha} \int_{t_{1}}^{t_{2}} \frac{\left(t_{1}-s\right)^{\alpha-m-1}}{\Gamma(\alpha-m)}\left|f\left(s, y(s), y^{\prime}(s), y^{\prime \prime}(s), D_{0^{+}}^{n} y(s)\right)\right| d s \\
& +t_{1}^{2+m-\alpha} \int_{0}^{t_{1}} \frac{\left|\left(t_{1}-s\right)^{\alpha-m-1}-\left(t_{2}-s\right)^{\alpha-m-1}\right|}{\Gamma(\alpha-m)} \times\left|f\left(s, y(s), y^{\prime}(s), y^{\prime \prime}(s), D_{0^{+}}^{n} y(s)\right)\right| d s
\end{aligned}
$$




$$
\begin{aligned}
& \leq M_{r}\left[\left|t_{2}^{2+m-\alpha}-t_{1}^{2+m-\alpha}\right| \int_{0}^{t_{2}} \frac{\left(t_{2}-s\right)^{\alpha-m-1}}{\Gamma(\alpha-m)} s^{k_{1}}(1-s)^{\sigma_{1}} d s\right. \\
& +t_{1}^{2+m-\alpha} \int_{t_{1}}^{t_{2}} \frac{\left(t_{1}-s\right)^{\alpha-m-1}}{\Gamma(\alpha-m)} s^{k_{1}}(1-s)^{\sigma_{1}} d s \\
& \left.+t_{1}^{2+m-\alpha} \int_{0}^{t_{1}} \frac{\left(t_{1}-s\right)^{\alpha-m-1}-\left(t_{2}-s\right)^{\alpha-m-1}}{\Gamma(\alpha-m)} s^{k_{1}}(1-s)^{\sigma_{1}} d s\right] \\
& \leq M_{r}\left[\left|t_{2}^{2+m-\alpha}-t_{1}^{2+m-\alpha}\right| t_{2}^{\alpha+\sigma_{1}+k_{1}-3} \int_{0}^{1} \frac{(1-w)^{\alpha+\sigma_{1}-m-1}}{\Gamma(\alpha-m)} w^{k_{1}} d w\right. \\
& +t_{1}^{2+m-\alpha} t_{2}^{\alpha+\sigma_{1}+k_{1}-m} \int_{t_{1} / t_{2}}^{1} \frac{(1-w)^{\alpha+\sigma_{1}-m-1}}{\Gamma(\alpha-m)} w^{k_{1}} d w \\
& +t_{1}^{2+m-\alpha} \int_{0}^{t_{1}} \frac{\left(t_{1}-s\right)^{\alpha-m-1}}{\Gamma(\alpha-m)} s^{k_{1}}\left(t_{1}-s\right)^{\sigma_{1}} d s \\
& \left.-t_{1}^{2+m-\alpha} \int_{0}^{t_{1}} \frac{\left(t_{2}-s\right)^{\alpha-m-1}}{\Gamma(\alpha-m)} s^{k_{1}}\left(t_{1}-s\right)^{\sigma_{1}} d s\right] \\
& \leq M_{r}\left[\left|t_{2}^{2+m-\alpha}-t_{1}^{2+m-\alpha}\right| \max \left\{e^{\alpha+\sigma_{1}+k_{1}-m}, d^{\alpha+\sigma_{1}+k_{1}-m}\right\}\right. \\
& \times \frac{\mathbf{B}\left(\alpha+\sigma_{1}-m, k_{1}+1\right)}{\Gamma(\alpha-m)} \\
& +\max \left\{e^{\alpha+\sigma_{1}+k_{1}-m}, d^{\alpha+\sigma_{1}+k_{1}-m}\right\} \int_{t_{1} / t_{2}}^{1} \frac{(1-w)^{\alpha+\sigma_{1}-m-1}}{\Gamma(\alpha-m)} w^{k_{1}} d w \\
& +t_{1}^{2+m-\alpha} t_{1}^{\alpha+\sigma_{1}+k_{1}-m} \int_{0}^{1} \frac{(1-w)^{\alpha-m-1}}{\Gamma(\alpha-m)} w^{k_{1}} d w \\
& \left.-t_{1}^{2+m-\alpha} \int_{0}^{t_{1}} \frac{\left(t_{2}-s\right)^{\alpha-m-1}}{\Gamma(\alpha-m)} s^{k_{1}}\left(t_{2}-s\right)^{\sigma_{1}} d s\right] \\
& \leq M_{r}\left[\left|t_{2}^{2+m-\alpha}-t_{1}^{2+m-\alpha}\right| \max \left\{e^{\alpha+\sigma_{1}+k_{1}-m}, d^{\alpha+\sigma_{1}+k_{1}-m}\right\}\right. \\
& \times \frac{\mathbf{B}\left(\alpha+\sigma_{1}-m, k_{1}+1\right)}{\Gamma(\alpha-m)} \\
& +\max \left\{e^{\alpha+\sigma_{1}+k_{1}-m}, d^{\alpha+\sigma_{1}+k_{1}-m}\right\} \int_{t_{1} / t_{2}}^{1} \frac{(1-w)^{\alpha+\sigma_{1}-m-1}}{\Gamma(\alpha-m)} w^{k_{1}} d w \\
& +t_{1}^{2+m-\alpha} t_{1}^{\alpha+\sigma_{1}+k_{1}-m} \int_{0}^{1} \frac{(1-w)^{\alpha+\sigma_{1}-m-1}}{\Gamma(\alpha-m)} w^{k_{1}} d w \\
& \left.-t_{1}^{2+m-\alpha} t_{2}^{\alpha+\sigma_{1}+k_{1}-m} \int_{0}^{t_{1} / t_{2}} \frac{(1-w)^{\alpha+\sigma_{1}-m-1}}{\Gamma(\alpha-m)} w^{k_{1}} d w\right]
\end{aligned}
$$




$$
\begin{aligned}
& \leq M_{r}\left[\left|t_{2}^{2+m-\alpha}-t_{1}^{2+m-\alpha}\right| \max \left\{e^{\alpha+\sigma_{1}+k_{1}-m}, d^{\alpha+\sigma_{1}+k_{1}-m}\right\}\right. \\
& \times \frac{\mathbf{B}\left(\alpha+\sigma_{1}-m, k_{1}+1\right)}{\Gamma(\alpha-m)} \\
&+\max \left\{e^{\alpha+\sigma_{1}+k_{1}-m}, d^{\alpha+\sigma_{1}+k_{1}-m}\right\} \int_{t_{1} / t_{2}}^{1} \frac{(1-w)^{\alpha+\sigma_{1}-m-1}}{\Gamma(\alpha-m)} w^{k_{1}} d w \\
&+t_{1}^{2+m-\alpha} t_{1}^{\alpha+\sigma_{1}+k_{1}-m} \int_{t_{1} / t_{2}}^{1} \frac{(1-w)^{\alpha+\sigma_{1}-m-1}}{\Gamma(\alpha-m)} w^{k_{1}} d w \\
&\left.+t_{1}^{2+m-\alpha}\left|t_{1}^{\alpha+\sigma_{1}+k_{1}-m}-t_{2}^{\alpha+\sigma_{1}+k_{1}-m}\right| \int_{0}^{t_{1} / t_{2}} \frac{(1-w)^{\alpha+\sigma_{1}-m-1}}{\Gamma(\alpha-m)} w^{k_{1}} d w\right] .
\end{aligned}
$$

We consider two cases:

CASE 1: $\alpha+\sigma_{1}+k_{1}-m>0$. We have

$$
\begin{aligned}
& \mid t_{1}^{2+m-\alpha} \int_{0}^{t_{1}} \frac{\left(t_{1}-s\right)^{\alpha-m-1}}{\Gamma(\alpha-m)} f\left(s, y(s), y^{\prime}(s), y^{\prime \prime}(s), D_{0^{+}}^{n} y(s)\right) d s \\
& -t_{2}^{2+m-\alpha} \int_{0}^{t_{2}} \frac{\left(t_{2}-s\right)^{\alpha-m-1}}{\Gamma(\alpha-m)} f\left(s, y(s), y^{\prime}(s), y^{\prime \prime}(s), D_{0^{+}}^{n} y(s)\right) d s \\
& \leq M_{r}\left[\left|t_{2}^{2+m-\alpha}-t_{1}^{2+m-\alpha}\right| \max \left\{e^{\alpha+\sigma_{1}+k_{1}-m}, f^{\alpha+\sigma_{1}+k_{1}-m}\right\}\right. \\
& \times \frac{\mathbf{B}\left(\alpha+\sigma_{1}-m, k_{1}+1\right)}{\Gamma(\alpha-m)} \\
& +\max \left\{e^{\alpha+\sigma_{1}+k_{1}-m}, d^{\alpha+\sigma_{1}+k_{1}-m}\right\} \int_{t_{1} / t_{2}}^{1} \frac{(1-w)^{\alpha+\sigma_{1}-m-1}}{\Gamma(\alpha-m)} w^{k_{1}} d w \\
& +\int_{t_{1} / t_{2}}^{1} \frac{(1-w)^{\alpha+\sigma_{1}-m-1}}{\Gamma(\alpha-m)} w^{k_{1}} d w \\
& \left.+\left|t_{1}^{\alpha+\sigma_{1}+k_{1}-m}-t_{2}^{\alpha+\sigma_{1}+k_{1}-m}\right| \int_{0}^{1} \frac{(1-w)^{\alpha+\sigma_{1}-m-1}}{\Gamma(\alpha-m)} w^{k_{1}} d w\right] .
\end{aligned}
$$

As $t_{1} \rightarrow t_{2}$, the right-hand side tends to zero uniformly. So

$$
\left|t_{1}^{2+m-\alpha} D_{0^{+}}^{m}\left(T_{1} y\right)\left(t_{1}\right)-t_{2}^{2+m-\alpha} D_{0^{+}}^{m}\left(T_{1} y\right)\left(t_{2}\right)\right| \rightarrow 0
$$

$$
\text { uniformly as } t_{1} \rightarrow t_{2} \text { on }[e, d] \subset(0,1] \text {. }
$$


CASE 2: $\alpha+\sigma_{1}+k_{1}-m \leq 0$. We have

$$
\begin{aligned}
& \mid t_{1}^{2+m-\alpha} \int_{0}^{t_{1}} \frac{\left(t_{1}-s\right)^{\alpha-m-1}}{\Gamma(\alpha-m)} f\left(s, y(s), y^{\prime}(s), y^{\prime \prime}(s), D_{0^{+}}^{n} y(s)\right) d s \\
& \quad-t_{2}^{2+m-\alpha} \int_{0}^{t_{2}} \frac{\left(t_{2}-s\right)^{\alpha-m-1}}{\Gamma(\alpha-m)} f\left(s, y(s), y^{\prime}(s), y^{\prime \prime}(s), D_{0^{+}}^{n} y(s)\right) d s \mid \\
& \leq M_{r}\left[\left|t_{2}^{2+m-\alpha}-t_{1}^{2+m-\alpha}\right| \max \left\{e^{\alpha+\sigma_{1}+k_{1}-m}, d^{\alpha+\sigma_{1}+k_{1}-m}\right\}\right. \\
& \quad+\max \left\{e^{\alpha+\sigma_{1}+k_{1}-m}, f^{\alpha+\sigma_{1}+k_{1}-m}\right\} \int_{t_{1} / t_{2}}^{1} \frac{(1-w)^{\alpha+\sigma_{1}-m-1}}{\Gamma(\alpha-m)} w^{k_{1}} d w \\
& \left.\quad+\int_{t_{1} / t_{2}}^{1} \frac{(1-w)^{\alpha+\sigma_{1}-m-1}}{\Gamma(\alpha-m)} w^{k_{1}} d w\right] .
\end{aligned}
$$

As $t_{1} \rightarrow t_{2}$, the right-hand side tends to zero uniformly. Hence (17) holds.

Furthermore,

$$
\begin{aligned}
& \mid t^{2+m-\alpha} D_{0^{+}}^{m}\left(T_{1} y\right)(t)-\left(-\frac{1}{\Gamma(\alpha-m-1)} \int_{0}^{1} f\left(s, y(s), y^{\prime}(s), y^{\prime \prime}(s), D_{0^{+}}^{n} y(s)\right) d s\right. \\
& \left.\quad+\frac{1}{\Gamma(\alpha-m-1)} \int_{0}^{1}(1-s) f\left(s, y(s), y^{\prime}(s), y^{\prime \prime}(s), D_{0^{+}}^{n} y(s)\right) d s\right) \mid \\
& \leq t^{2+m-\alpha} \int_{0}^{t} \frac{(t-s)^{\alpha-m-1}}{\Gamma(\alpha-m)}\left|f\left(s, y(s), y^{\prime}(s), y^{\prime \prime}(s), D_{0^{+}}^{n} y(s)\right)\right| d s \\
& \quad+\frac{1}{\Gamma(\alpha-m)} t \int_{0}^{1}\left|f\left(s, y(s), y^{\prime}(s), y^{\prime \prime}(s), D_{0^{+}}^{n} y(s)\right)\right| d s \\
& \leq M_{r} t^{2+m-\alpha} \int_{0}^{t} \frac{(t-s)^{\alpha-m-1}}{\Gamma(\alpha-m)} s^{k_{1}}(1-s)^{\sigma_{1}} d s+\frac{M_{r}}{\Gamma(\alpha-m)} t \int_{0}^{1} s^{k_{1}}(1-s)^{\sigma_{1}} d s \\
& \leq M_{r} t^{2+\sigma_{1}+k_{1}} \int_{0}^{1} \frac{(1-w)^{\alpha+\sigma_{1}-m}}{\Gamma(\alpha-m)} w^{k_{1}} d w+\frac{M_{r}}{\Gamma(\alpha-m)} t \mathbf{B}\left(\sigma_{1}+1, k_{1}+1\right) .
\end{aligned}
$$

It follows that

$$
t^{2+m-\alpha} D_{0^{+}}^{m}\left(T_{1} y\right)(t) \text { is uniformly convergent as } t \rightarrow 0 .
$$


From (17) and (18), we deduce that $\left\{t^{2+m-\alpha} D_{0^{+}}^{m}\left(T_{1} y\right)(t): y \in \Omega\right\}$ is equicontinuous on $(0,1]$.

Therefore, $T_{1} \Omega$ is relatively compact.

From the above discussion, $T_{1}$ is completely continuous. The proof is complete.

\section{LEMMA 2.4. Suppose that}

(B2) $g(t, x, y, z, w)$ is continuous on $(0,1) \times \mathbb{R}^{4}$ and for each $r>0$ there exist $N_{r}>0, k_{2}>-1, \sigma_{2} \leq 0$ such that $2+\sigma_{2}+k_{2}>0$ and

$$
\left|g\left(t, t^{\alpha-2} x, t^{\alpha-3} y, t^{\alpha-4} z, t^{\alpha-m-2} w\right)\right| \leq N_{r} t^{k_{2}}(1-t)^{\sigma_{2}}
$$

for all $t \in(0,1)$ and $|x|,|y|,|z|,|w| \leq r$.

Then $T_{2}: X \rightarrow Y$ is completely continuous.

Proof. The proof is similar to that of Lemma 2.3 and is omitted.

REMARK 2.6. Suppose that (B1) and (B2) hold. It follows from Lemmas 2.3 and 2.4 that $T: X \times Y \rightarrow X \times Y$ is completely continuous.

3. Main results. In this section, we prove our main results. We need assumptions imposed on $f, g$ given in Section 1 and the following assumptions:

(B3) $\psi \in C^{0}(0,1)$ and there exist $c_{0}>-1,-1<d_{0} \leq 0\left(2+c_{0}+d_{0}>0\right)$ and $N_{0}>0$ such that $|\psi(t)| \leq N_{0} t^{c_{0}}(1-t)^{d_{0}}$ for all $t \in(0,1)$.

(B4) $\phi \in C^{0}(0,1)$ and there exist $a_{0}>-1,-1<b_{0} \leq 0\left(2+a_{0}+b_{0}>0\right)$ and $M_{0}>0$ such that $|\phi(t)| \leq M_{0} t^{a_{0}}(1-t)^{b_{0}}$ for all $t \in(0,1)$.

(B5) $f:(0,1) \times \mathbb{R}^{4} \rightarrow[0, \infty)$ is continuous and there exist $a_{i}>-1$, $b_{i} \leq 0(i=1, \ldots, \omega)$ with $2+a_{i}+b_{i}>0, \tau_{i 1}, \tau_{i 2}, \tau_{i 3}, \tau_{i 4} \geq 0$ $(i=1, \ldots, \omega)$ and $A_{i} \geq 0(i=1, \ldots, \omega)$ such that

$$
\begin{aligned}
\left|f\left(t, t^{\beta-2} x, t^{\beta-3} y, t^{\beta-4} z, t^{\beta-n-2} w\right)-\psi(t)\right| & \\
\leq & \leq \sum_{i=1}^{\omega} A_{i} t^{a_{i}}(1-t)^{b_{i}}|x|^{\tau_{i 1}}|y|^{\tau_{i 2}}|z|^{\tau_{i 3}}|w|^{\tau_{i 4}}
\end{aligned}
$$

for all $t \in(0,1)$ and $x, y, z, w \in \mathbb{R}$.

(B6) $g:(0,1) \times \mathbb{R}^{4} \rightarrow[0, \infty)$ is continuous and there exist $c_{i}>-1$, $d_{i} \leq 0(i=1, \ldots, \omega)$ with $2+c_{i}+d_{i}>0, \delta_{i 1}, \delta_{i 2}, \delta_{i 3}, \delta_{i 4} \geq 0$ $(i=1, \ldots, \omega)$ and $B_{i} \geq 0(i=1, \ldots, \omega)$ such that

$$
\begin{aligned}
\left|g\left(t, t^{\alpha-2} x, t^{\alpha-3} y, t^{\alpha-4} x, t^{\alpha-m-2} y\right)-\phi(t)\right| & \\
& \leq \sum_{i=1}^{\omega} B_{i} t^{c_{i}}(1-t)^{d_{i}}|x|^{\delta_{i 1}}|y|^{\delta_{i 2}}|z|^{\delta_{i 3}}|w|^{\delta_{i 4}}
\end{aligned}
$$

for all $t \in(0,1)$ and $x, y, z, w \in \mathbb{R}$. 
Set

$$
\begin{aligned}
& \tau_{i}=\tau_{i 1}+\tau_{i 2}+\tau_{i 3}+\tau_{i 4}(i=1, \ldots, \omega), \quad \tau=\max \left\{\tau_{i}: i=1, \ldots, \omega\right\}, \\
& \delta_{i}=\delta_{i 1}+\delta_{i 2}+\delta_{i 3}+\delta_{i 4}(i=1, \ldots, \omega), \quad \delta=\max \left\{\delta_{i}: i=1, \ldots, \omega\right\}, \\
& \Psi(t)=\int_{0}^{t} \frac{(t-s)^{\beta-1}}{\Gamma(\beta)} \psi(s) d s-\left[\frac{t^{\beta-1}}{\Gamma(\beta)}-\frac{t^{\beta-2}}{\Gamma(\beta-1)}\right] \int_{0}^{1} \psi(s) d s \\
& -\frac{t^{\beta-2}}{\Gamma(\beta-1)} \int_{0}^{1}(1-s) \psi(s) d s \\
& \Phi(t)=\int_{0}^{t} \frac{(t-s)^{\alpha-1}}{\Gamma(\alpha)} \phi(s) d s-\left[\frac{t^{\alpha-1}}{\Gamma(\alpha)}-\frac{t^{\alpha-2}}{\Gamma(\alpha-1)}\right] \int_{0}^{1} \phi(s) d s \\
& -\frac{t^{\alpha-2}}{\Gamma(\alpha-1)} \int_{0}^{1}(1-s) \phi(s) d s \\
& P_{i}=\frac{\mathbf{B}\left(\alpha+b_{i}, a_{i}+1\right)}{\Gamma(\alpha)}+\left[\frac{1}{\Gamma(\alpha)}+\frac{1}{\Gamma(\alpha-1)}\right] \mathbf{B}\left(b_{i}+1, a_{i}+1\right) \\
& +\frac{\mathbf{B}\left(b_{i}+2, a_{i}+1\right)}{\Gamma(\alpha-1)} \\
& Q_{i}=\frac{\mathbf{B}\left(\alpha+b_{i}-1, a_{i}+1\right)}{\Gamma(\alpha-1)}+\left[\frac{1}{\Gamma(\alpha-1)}+\frac{1}{\Gamma(\alpha-2)}\right] \mathbf{B}\left(b_{i}+1, a_{i}+1\right) \\
& +\frac{\mathbf{B}\left(b_{i}+2, a_{i}+1\right)}{\Gamma(\alpha-2)} \\
& R_{i}=\frac{\mathbf{B}\left(\alpha+b_{i}-2, a_{i}+1\right)}{\Gamma(\alpha-2)}+\left[\frac{1}{\Gamma(\alpha-2)}+\frac{1}{\Gamma(\alpha-3)}\right] \mathbf{B}\left(b_{i}+1, a_{i}+1\right) \\
& +\frac{\mathbf{B}\left(b_{i}+2, a_{i}+1\right)}{\Gamma(\alpha-3)} \\
& W_{i}=\frac{\mathbf{B}\left(\alpha+b_{i}-m, a_{i}+1\right)}{\Gamma(\alpha-m)}+\left[\frac{1}{\Gamma(\alpha-m)}+\frac{1}{\Gamma(\alpha-m-1)}\right] \mathbf{B}\left(b_{i}+1, a_{i}+1\right) \\
& +\frac{\mathbf{B}\left(b_{i}+2, a_{i}+1\right)}{\Gamma(\alpha-m-1)}, \\
& \Xi_{i}=\max \left\{P_{i}, Q_{i}, R_{i}, W_{i}\right\}, \quad i=1, \ldots, \omega,
\end{aligned}
$$

and

$$
\begin{aligned}
\bar{P}_{i}= & \frac{\mathbf{B}\left(\beta+d_{i}, c_{i}+1\right)}{\Gamma(\beta)}+\left[\frac{1}{\Gamma(\beta)}+\frac{1}{\Gamma(\beta-1)}\right] \mathbf{B}\left(d_{i}+1, c_{i}+1\right) \\
& +\frac{\mathbf{B}\left(d_{i}+2, c_{i}+1\right)}{\Gamma(\beta-1)}
\end{aligned}
$$




$$
\begin{aligned}
\bar{Q}_{i}= & \frac{\mathbf{B}\left(\beta+d_{i}-1, c_{i}+1\right)}{\Gamma(\beta-1)}+\left[\frac{1}{\Gamma(\beta-1)}+\frac{1}{\Gamma(\beta-2)}\right] \mathbf{B}\left(d_{i}+1, c_{i}+1\right) \\
& +\frac{\mathbf{B}\left(d_{i}+2, c_{i}+1\right)}{\Gamma(\beta-2)}, \\
\bar{R}_{i}= & \frac{\mathbf{B}\left(\beta+d_{i}-2, c_{i}+1\right)}{\Gamma(\beta-2)}+\left[\frac{1}{\Gamma(\beta-2)}+\frac{1}{\Gamma(\beta-3)}\right] \mathbf{B}\left(d_{i}+1, c_{i}+1\right) \\
& +\frac{\mathbf{B}\left(d_{i}+2, c_{i}+1\right)}{\Gamma(\beta-3)}, \\
\bar{W}_{i}= & \frac{\mathbf{B}\left(\beta+d_{i}-n, c_{i}+1\right)}{\Gamma(\beta-n)}+\left[\frac{1}{\Gamma(\beta-n)}+\frac{1}{\Gamma(\beta-n-1)}\right] \mathbf{B}\left(d_{i}+1, c_{i}+1\right) \\
& +\frac{\mathbf{B}\left(d_{i}+2, c_{i}+1\right)}{\Gamma(\beta-n-1)}, \quad i=1, \ldots, \omega .
\end{aligned}
$$

TheOREM 3.1. Suppose that (B3), (B4), (B5) and (B6) hold. Then $\mathrm{BVP}(1)$ has at least one positive solution if

(i) $\tau \delta<1$, or

(ii) $\tau \delta=1$ with

$$
\sum_{i=1}^{\omega} B_{i} \bar{\Xi}_{i}\|\Phi\|^{\delta_{i}-\delta}\left[\sum_{i=1}^{\omega} A_{i} \Xi_{i}\|\Psi\|^{\tau_{i}-\tau}\right]^{1 / \tau}<1,
$$

or

(iii) $\tau \delta>1$ with

$$
\frac{\left(\frac{\|\Phi\|}{\delta \tau-1}+\|\Phi\|\right)^{\delta}}{\left(\frac{\|\Phi\|}{\delta \tau-1}\right)^{1 / \tau}} \sum_{i=1}^{\omega} B_{i} \bar{\Xi}_{i}\|\Phi\|^{\delta_{i}-\delta}+\frac{\|\Psi\|}{\left(\frac{\|\Phi\|}{\delta \tau-1}\right)^{1 / \tau}} \leq \frac{1}{\left[\sum_{i=1}^{\omega} A_{i} \Xi_{i}\|\Psi\| \tau_{i}-\tau\right]^{1 / \tau}} .
$$

Proof. It is easy to show that (B5) and (B6) imply (B1) and (B2). Let the Banach space $X \times Y$ and the operator $T$ on $X \times Y$ be as defined in Section 2. By Lemmas 2.3 and 2.4, $T: X \times Y \rightarrow X \times Y$ is well defined and completely continuous. By the assumptions on $f, g$ in Section 1, together with Remark 2.5, we know that $(x, y) \in X \times Y$ is a nonnegative solution of $\operatorname{BVP}(1)$ if and only if $(x, y) \in X \times Y$ is a fixed point of $T$. If there exists $t_{0} \in(0,1)$ such that $\left[x\left(t_{0}\right)\right]^{2}+\left[y\left(t_{0}\right)\right]^{2}=0$, we have $x\left(t_{0}\right)=y\left(t_{0}\right)=0$. Then similarly to Remark 2.2, we get

$$
\begin{aligned}
& {\left[g\left(t, t^{\alpha-2} x, t^{\alpha-3} y, t^{\alpha-4} z, t^{\alpha-m-2} w\right)\right]^{2}} \\
& +\left[f\left(t, t^{\beta-2} x, t^{\beta-3} y, t^{\beta-4} z, t^{\beta-m-2} w\right)\right]^{2} \equiv 0
\end{aligned}
$$

on some subinterval $(0, c]$ with $c \in(0,1)$, contradicting the assumptions of Section 1. So $(x, y)$ is a positive solution of $\operatorname{BVP}(1)$ if $(x, y)$ is a fixed point of $T$. 
It is easy to see from (B3), (B4) and Lemmas 2.1 and 2.2 that $\Psi \in Y$ and $\Phi \in X$. By direct computation, we have

$$
\begin{aligned}
& \Psi^{\prime}(t)=\int_{0}^{t} \frac{(t-s)^{\beta-2}}{\Gamma(\beta-1)} \psi(s) d s-\left[\frac{t^{\beta-2}}{\Gamma(\beta-1)}-\frac{t^{\beta-3}}{\Gamma(\beta-2)}\right] \int_{0}^{1} \psi(s) d s \\
& -\frac{t^{\beta-3}}{\Gamma(\beta-2)} \int_{0}^{1}(1-s) \psi(s) d s, \\
& \Psi^{\prime \prime}(t)=\int_{0}^{t} \frac{(t-s)^{\beta-3}}{\Gamma(\beta-2)} \psi(s) d s-\left[\frac{t^{\beta-3}}{\Gamma(\beta-2)}-\frac{t^{\beta-4}}{\Gamma(\beta-3)}\right] \int_{0}^{1} \psi(s) d s \\
& -\frac{t^{\beta-4}}{\Gamma(\beta-3)} \int_{0}^{1}(1-s) \psi(s) d s, \\
& D_{0^{+}}^{n} \Psi(t)=\int_{0}^{t} \frac{(t-s)^{\beta-n-1}}{\Gamma(\beta-n)} \psi(s) d s \\
& -\left[\frac{t^{\beta-n-1}}{\Gamma(\beta-n)}-\frac{t^{\beta-n-2}}{\Gamma(\beta-n-1)}\right] \int_{0}^{1} \psi(s) d s \\
& -\frac{t^{\beta-n-2}}{\Gamma(\beta-n-1)} \int_{0}^{1}(1-s) \psi(s) d s, \\
& \Phi^{\prime}(t)=\int_{0}^{t} \frac{(t-s)^{\alpha-2}}{\Gamma(\alpha-1)} \phi(s) d s-\left[\frac{t^{\alpha-2}}{\Gamma(\alpha-1)}-\frac{t^{\alpha-3}}{\Gamma(\alpha-2)}\right] \int_{0}^{1} \phi(s) d s \\
& -\frac{t^{\alpha-3}}{\Gamma(\alpha-2)} \int_{0}^{1}(1-s) \phi(s) d s, \\
& \Phi^{\prime \prime}(t)=\int_{0}^{t} \frac{(t-s)^{\alpha-3}}{\Gamma(\alpha-2)} \phi(s) d s-\left[\frac{t^{\alpha-3}}{\Gamma(\alpha-2)}-\frac{t^{\alpha-4}}{\Gamma(\alpha-3)}\right] \int_{0}^{1} \phi(s) d s \\
& -\frac{t^{\alpha-4}}{\Gamma(\alpha-3)} \int_{0}^{1}(1-s) \phi(s) d s \\
& D_{0^{+}}^{m} \Phi(t)=\int_{0}^{t} \frac{(t-s)^{\alpha-m-1}}{\Gamma(\alpha-m)} \phi(s) d s-\left[\frac{t^{\alpha-m-1}}{\Gamma(\alpha-m)}-\frac{t^{\alpha-m-2}}{\Gamma(\alpha-m-1)}\right] \int_{0}^{1} \phi(s) d s \\
& -\frac{t^{\alpha-m-2}}{\Gamma(\alpha-m-1)} \int_{0}^{1}(1-s) \phi(s) d s .
\end{aligned}
$$

For $r_{1}, r_{2}>0$, denote

$$
\Omega_{r_{1}, r_{2}}=\left\{(x, y) \in X \times Y:\|y-\Psi\| \leq r_{2},\|x-\Phi\| \leq r_{1}\right\} .
$$


One sees, for $(x, y) \in \Omega_{r_{1}, r_{2}}$, that

$$
\begin{gathered}
\|x\| \leq\|x-\Psi\|+\|\Psi\| \leq r_{1}+\|\Psi\|, \\
\|y\| \leq\|y-\Phi\|+\|\Phi\| \leq r_{2}+\|\Phi\| .
\end{gathered}
$$

Hence for $(x, y) \in \Omega_{r}$, we have

$$
\begin{aligned}
& \left|f\left(t, y(t), y^{\prime}(t), y^{\prime \prime}(t), D_{0^{+}}^{n} y(t)\right)-\psi(t)\right| \\
& \leq \sum_{i=1}^{\omega} A_{i} t^{a_{i}}(1-t)^{b_{i}}\left|t^{2-\beta} y(t)\right|^{\tau_{i 1} \mid}\left|t^{3-\beta} y^{\prime}(t)\right|^{\tau_{i 2}}\left|t^{4-\beta} y^{\prime \prime}(t)\right|^{\tau_{i 3}}\left|t^{2+n-\beta} D_{0^{+}}^{n} y(t)\right|^{\tau_{i 4}} \\
& \leq \sum_{i=1}^{\omega} A_{i} t^{a_{i}}(1-t)^{b_{i}}\left[r_{2}+\|\Psi\|\right]^{\tau_{i 1}+\tau_{i 2}+\tau_{i 3}+\tau_{i 4}} \\
& =\sum_{i=1}^{\omega} A_{i} t^{a_{i}}(1-t)^{b_{i}}\left[r_{2}+\|\Psi\|\right]^{\tau_{i}}
\end{aligned}
$$

and

$$
\left|g\left(t, x(t), x^{\prime}(t), x^{\prime \prime}(t), D_{0^{+}}^{m} x(t)\right)-\phi(t)\right| \leq \sum_{i=1}^{\omega} B_{i} t^{c_{i}}(1-t)^{d_{i}}\left[r_{1}+\|\Phi\|\right]^{\delta_{i}} .
$$

Firstly, we have

$$
\begin{aligned}
t^{2-\alpha}\left|\left(T_{1} y\right)(t)-\Phi(t)\right| & \\
\leq & t^{2-\alpha} \int_{0}^{t} \frac{(t-s)^{\alpha-1}}{\Gamma(\alpha)}\left|f\left(s, y(s), y^{\prime}(s), y^{\prime \prime}(s), D_{0^{+}}^{n} y(s)\right)-\phi(s)\right| d s \\
& +\left[\frac{1}{\Gamma(\alpha)}+\frac{1}{\Gamma(\alpha-1)}\right] \int_{0}^{1}\left|f\left(s, y(s), y^{\prime}(s), y^{\prime \prime}(s), D_{0^{+}}^{n} y(s)\right)-\phi(s)\right| d s \\
& +\frac{1}{\Gamma(\alpha-1)} \int_{0}^{1}(1-s)\left|f\left(s, y(s), y^{\prime}(s), y^{\prime \prime}(s), D_{0^{+}}^{n} y(s)\right)-\phi(s)\right| d s \\
\leq & t^{2-\alpha} \int_{0}^{t} \frac{(t-s)^{\alpha-1}}{\Gamma(\alpha)} \sum_{i=1}^{m} A_{i} s^{a_{i}}(1-s)^{b_{i}}\left[r_{2}+\|\Psi\|\right]^{\tau_{i}} d s \\
& +\left[\frac{1}{\Gamma(\alpha)}+\frac{1}{\Gamma(\alpha-1)}\right] \int_{0}^{1} \sum_{i=1}^{m} A_{i} s^{a_{i}}(1-s)^{b_{i}}\left[r_{2}+\|\Psi\|\right]^{\tau_{i}} d s \\
& +\frac{1}{\Gamma(\alpha-1)} \int_{0}^{1}(1-s) \sum_{i=1}^{m} A_{i} s^{a_{i}}(1-s)^{b_{i}}\left[r_{2}+\|\Psi\|\right]^{\tau_{i}} d s
\end{aligned}
$$




$$
\begin{aligned}
& \leq t^{2-\alpha} \sum_{i=1}^{m} A_{i}\left[r_{2}+\|\Psi\|\right]^{\tau_{i}} \int_{0}^{t} \frac{(t-s)^{\alpha-1}}{\Gamma(\alpha)} s^{a_{i}}(t-s)^{b_{i}} d s \\
& +\left[\frac{1}{\Gamma(\alpha)}+\frac{1}{\Gamma(\alpha-1)}\right] \sum_{i=1}^{m} A_{i}\left[r_{2}+\|\Psi\|\right]^{\tau_{i}} \int_{0}^{1} s^{a_{i}}(1-s)^{b_{i}} d s \\
& +\frac{1}{\Gamma(\alpha-1)} \sum_{i=1}^{m} A_{i}\left[r_{2}+\|\Psi\|\right]^{\tau_{i}} \int_{0}^{1}(1-s) s^{a_{i}}(1-s)^{b_{i}} d s \\
& =t^{2-\alpha} \sum_{i=1}^{m} A_{i}\left[r_{2}+\|\Psi\|\right]^{\tau_{i}} t^{\alpha+a_{i}+b_{i}} \int_{0}^{1} \frac{(1-w)^{\alpha+b_{i}-1}}{\Gamma(\alpha)} w^{a_{i}} d w \\
& +\left[\frac{1}{\Gamma(\alpha)}+\frac{1}{\Gamma(\alpha-1)}\right] \sum_{i=1}^{m} A_{i}\left[r_{2}+\|\Psi\|\right]^{\tau_{i}} \mathbf{B}\left(b_{i}+1, a_{i}+1\right) \\
& +\frac{1}{\Gamma(\alpha-1)} \sum_{i=1}^{m} A_{i}\left[r_{2}+\|\Psi\|\right]^{\tau_{i}} \mathbf{B}\left(b_{i}+2, a_{i}+1\right) \\
& \leq \sum_{i=1}^{m} A_{i}\left[r_{2}+\|\Psi\|\right]^{\tau_{i}} \frac{\mathbf{B}\left(\alpha+b_{i}, a_{i}+1\right)}{\Gamma(\alpha)} \\
& +\left[\frac{1}{\Gamma(\alpha)}+\frac{1}{\Gamma(\alpha-1)}\right] \sum_{i=1}^{m} A_{i}\left[r_{2}+\|\Psi\|\right]^{\tau_{i}} \mathbf{B}\left(b_{i}+1, a_{i}+1\right) \\
& +\frac{1}{\Gamma(\alpha-1)} \sum_{i=1}^{m} A_{i}\left[r_{2}+\|\Psi\|\right]^{\tau_{i}} \mathbf{B}\left(b_{i}+2, a_{i}+1\right) \\
& =\sum_{i=1}^{\omega} A_{i} P_{i}\left[r_{2}+\|\Psi\|\right]^{\tau_{i}}
\end{aligned}
$$

It follows that

$$
\sup _{t \in(0,1]} t^{2-\alpha}\left|\left(T_{1} y\right)(t)-\Phi(t)\right| \leq \sum_{i=1}^{\omega} A_{i} P_{i}\left[r_{2}+\|\Psi\|\right]^{\tau_{i}} .
$$

Secondly,

$$
\begin{aligned}
& t^{3-\alpha}\left|\left(T_{1} y\right)^{\prime}(t)-\Phi^{\prime}(t)\right| \\
& \quad \leq \int_{0}^{t} \frac{(t-s)^{\alpha-2}}{\Gamma(\alpha-1)}\left|f\left(s, y(s), y^{\prime}(s), y^{\prime \prime}(s), D_{0^{+}}^{n} y(s)\right)-\phi(s)\right| d s \\
& \quad+\left[\frac{1}{\Gamma(\alpha-1)}+\frac{1}{\Gamma(\alpha-2)}\right] \int_{0}^{1}\left|f\left(s, y(s), y^{\prime}(s), y^{\prime \prime}(s), D_{0^{+}}^{n} y(s)\right)-\phi(s)\right| d s
\end{aligned}
$$




$$
\begin{aligned}
& +\frac{1}{\Gamma(\alpha-2)} \int_{0}^{1}(1-s)\left|f\left(s, y(s), y^{\prime}(s), y^{\prime \prime}(s), D_{0^{+}}^{n} y(s)\right)-\phi(s)\right| d s \\
\leq & \sum_{i=1}^{\omega} A_{i} Q_{i}\left[r_{2}+\|\Psi\|\right]^{\tau_{i}} .
\end{aligned}
$$

It follows that

$$
\sup _{t \in(0,1]} t^{3-\alpha}\left|\left(T_{1} y\right)^{\prime}(t)-\Phi^{\prime}(t)\right| \leq \sum_{i=1}^{\omega} A_{i} Q_{i}\left[r_{2}+\|\Psi\|\right]^{\tau_{i}} .
$$

Thirdly, we have similarly

$$
\sup _{t \in(0,1]} t^{4-\alpha}\left|\left(T_{1} y\right)^{\prime \prime}(t)-\Phi^{\prime \prime}(t)\right| \leq \sum_{i=1}^{\omega} A_{i} R_{i}\left[r_{2}+\|\Psi\|\right]^{\tau_{i}} .
$$

Finally,

$$
\sup _{t \in(0,1]} t^{2+m-\alpha}\left|D_{0^{+}}^{m}\left(T_{1} y\right)(t)-D_{0^{+}}^{m} \Phi(t)\right| \leq \sum_{i=1}^{\omega} A_{i} W_{i}\left[r_{2}+\|\Psi\|\right]^{\tau_{i}} .
$$

Thus (19)-(22) imply that

$$
\left\|T_{1} y-\Phi\right\| \leq \sum_{i=1}^{\omega} A_{i} \Xi_{i}\left[r_{2}+\|\Psi\|\right]^{\tau_{i}} \leq\left[r_{2}+\|\Psi\|\right]^{\tau} \sum_{i=1}^{\omega} A_{i} \Xi_{i}\|\Psi\|^{\tau_{i}-\tau} .
$$

Similarly we can prove that

$$
\left\|T_{2} x-\Psi\right\| \leq \sum_{i=1}^{\omega} B_{i} \bar{\Xi}_{i}\left[r_{1}+\|\Phi\|\right]^{\delta_{i}} \leq\left[r_{1}+\|\Phi\|\right]^{\delta} \sum_{i=1}^{\omega} B_{i} \bar{\Xi}_{i}\|\Phi\|^{\delta_{i}-\delta} .
$$

In order to apply Schauder's fixed point theorem, we will construct $\Omega_{r_{1}, r_{2}}$ such that $T\left(\Omega_{r_{1}, r_{2}}\right) \subseteq \Omega_{r_{1}, r_{2}}$. From (23) and (24), our purpose is to choose $r_{1}$ and $r_{2}$ such that

i.e.,

$$
\begin{gathered}
{\left[r_{2}+\|\Psi\|\right]^{\tau} \sum_{i=1}^{\omega} A_{i} \Xi_{i}\|\Psi\|^{\tau_{i}-\tau} \leq r_{1},} \\
{\left[r_{1}+\|\Phi\|\right]^{\delta} \sum_{i=1}^{\omega} B_{i} \bar{\Xi}_{i}\|\Phi\|^{\delta_{i}-\delta} \leq r_{2},}
\end{gathered}
$$

$$
\left[r_{1}+\|\Phi\|\right]^{\delta} \sum_{i=1}^{\omega} B_{i} \bar{\Xi}_{i}\|\Phi\|^{\delta_{i}-\delta} \leq r_{2} \leq \frac{r_{1}^{1 / \tau}}{\left[\sum_{i=1}^{\omega} A_{i} \Xi_{i}\|\Psi\|^{\tau_{i}-\tau}\right]^{1 / \tau}}-\|\Psi\|
$$

or

$$
\left[r_{2}+\|\Psi\|\right]^{\tau} \sum_{i=1}^{\omega} A_{i} \Xi_{i}\|\Psi\|^{\tau_{i}-\tau} \leq r_{1} \leq \frac{r_{2}^{1 / \delta}}{\left[\sum_{i=1}^{\omega} B_{i} \bar{\Xi}_{i}\|\Phi\|^{\delta_{i}-\delta}\right]^{1 / \delta}}-\|\Phi\| .
$$

We consider three cases: 
Case (i): $\tau \delta<1$. Since $\tau \delta<1$ implies that there exists $r_{1}>0$ sufficiently large such that

$$
\left[r_{1}+\|\Phi\|\right]^{\delta} \sum_{i=1}^{\omega} B_{i} \bar{\Xi}_{i}\|\Phi\|^{\delta_{i}-\delta}+\|\Psi\| \leq \frac{r_{1}^{1 / \tau}}{\left[\sum_{i=1}^{\omega} A_{i} \Xi_{i}\|\Psi\|^{\tau_{i}-\tau}\right]^{1 / \tau}},
$$

we can choose

$$
r_{2}=\left[r_{1}+\|\Phi\|\right]^{\delta} \sum_{i=1}^{\omega} B_{i} \bar{\Xi}_{i}\|\Phi\|^{\delta_{i}-\delta} .
$$

Then

$$
\left[r_{1}+\|\Phi\|\right]^{\delta} \sum_{i=1}^{\omega} B_{i} \bar{\Xi}_{i}\|\Phi\|^{\delta_{i}-\delta}=r_{2} \leq \frac{r_{1}^{1 / \tau}}{\left[\sum_{i=1}^{\omega} A_{i} \Xi_{i}\|\Psi\|^{\tau_{i}-\tau}\right]^{1 / \tau}}-\|\Psi\| .
$$

Set $\Omega_{r_{1}, r_{2}}=\left\{(x, y) \in X \times Y:\|y-\Psi\| \leq r_{2},\|x-\Phi\| \leq r_{1}\right\}$. From the above discussion,

$$
\begin{gathered}
\left\|T_{1} y-\Phi\right\| \leq\left[r_{2}+\|\Psi\|\right]^{\tau} \sum_{i=1}^{\omega} A_{i} \Xi_{i}\|\Psi\|^{\tau_{i}-\tau} \leq r_{1}, \\
\left\|T_{2} x-\Psi\right\| \leq\left[r_{1}+\|\Phi\|\right]^{\delta} \sum_{i=1}^{\omega} B_{i} \bar{\Xi}_{i}\|\Phi\|^{\delta_{i}-\delta} \leq r_{2} .
\end{gathered}
$$

Hence $T(x, y) \in \Omega_{r_{1}, r_{2}}$. By Schauder's fixed point theorem, $T$ has at least one fixed point $(x, y) \in \Omega_{r_{1}, r_{2}}$. Then $(x, y)$ is a solution of $\operatorname{BVP}(1)$.

Case (ii): $\tau \delta=1$. Since

$$
\sum_{i=1}^{\omega} B_{i} \bar{\Xi}_{i}\|\Phi\|^{\delta_{i}-\delta}<\frac{1}{\left[\sum_{i=1}^{\omega} A_{i} \Xi_{i}\|\Psi\|^{\tau_{i}-\tau}\right]^{1 / \tau}},
$$

there exists $r_{1}>0$ sufficiently large such that

$$
\left[r_{1}+\|\Phi\|\right]^{\delta} \sum_{i=1}^{\omega} B_{i} \bar{\Xi}_{i}\|\Phi\|^{\delta_{i}-\delta}+\|\Psi\| \leq \frac{r_{1}^{1 / \tau}}{\left[\sum_{i=1}^{\omega} A_{i} \Xi_{i}\|\Psi\|^{\tau_{i}-\tau}\right]^{1 / \tau}} .
$$

Choose

$$
r_{2}=\left[r_{1}+\|\Phi\|\right]^{\delta} \sum_{i=1}^{\omega} B_{i} \bar{\Xi}_{i}\|\Phi\|^{\delta_{i}-\delta} .
$$

Then

$$
\left[r_{1}+\|\Phi\|\right]^{\delta} \sum_{i=1}^{\omega} B_{i} \bar{\Xi}_{i}\|\Phi\|^{\delta_{i}-\delta}=r_{2} \leq \frac{r_{1}^{1 / \tau}}{\left[\sum_{i=1}^{\omega} A_{i} \Xi_{i}\|\Psi\|^{\tau_{i}-\tau}\right]^{1 / \tau}}-\|\Psi\| .
$$

Set $\Omega_{r_{1}, r_{2}}=\left\{(x, y) \in X \times Y:\|y-\Psi\| \leq r_{2},\|x-\Phi\| \leq r_{1}\right\}$. From the above 
discussion,

$$
\begin{aligned}
& \left\|T_{1} y-\Phi\right\| \leq\left[r_{2}+\|\Psi\|\right]^{\tau} \sum_{i=1}^{\omega} A_{i} \Xi_{i}\|\Psi\|^{\tau_{i}-\tau} \leq r_{1}, \\
& \left\|T_{2} x-\Psi\right\| \leq\left[r_{1}+\|\Phi\|\right]^{\delta} \sum_{i=1}^{\omega} B_{i} \bar{\Xi}_{i}\|\Phi\|^{\delta_{i}-\delta} \leq r_{2} .
\end{aligned}
$$

Hence $T(x, y) \in \Omega_{r_{1}, r_{2}}$. By Schauder's fixed point theorem, $T$ has at least one fixed point $(x, y) \in \Omega_{r_{1}, r_{2}}$. Then $(x, y)$ is a solution of $\operatorname{BVP}(1)$.

CASE (iii): $\tau \delta>1$. Choose

$$
r_{1}=\frac{\|\Phi\|}{\delta \tau-1} .
$$

It is easy to show from the inequality in Theorem 3.1(iii) that

$$
\frac{\left(r_{1}+\|\Phi\|\right)^{\delta}}{r_{1}^{1 / \tau}} \sum_{i=1}^{\omega} B_{i} \bar{\Xi}_{i}\|\Phi\|^{\delta_{i}-\delta}+\frac{\|\Psi\|}{r_{1}^{1 / \tau}} \leq \frac{1}{\left[\sum_{i=1}^{\omega} A_{i} \Xi_{i}\|\Psi\|^{\tau_{i}-\tau}\right]^{1 / \tau}} .
$$

Then

$$
\left(r_{1}+\|\Phi\|\right)^{\delta} \sum_{i=1}^{\omega} B_{i} \bar{\Xi}_{i}\|\Phi\|^{\delta_{i}-\delta}+\|\Psi\| \leq \frac{r_{1}^{1 / \tau}}{\left[\sum_{i=1}^{\omega} A_{i} \Xi_{i}\|\Psi\|^{\tau_{i}-\tau}\right]^{1 / \tau}} .
$$

Choose

$$
r_{2}=\left(r_{1}+\|\Phi\|\right)^{\delta} \sum_{i=1}^{\omega} B_{i} \bar{\Xi}_{i}\|\Phi\|^{\delta_{i}-\delta} .
$$

We also have (25). Set $\Omega_{r_{1}, r_{2}}=\left\{(x, y) \in X \times Y:\|y-\Psi\| \leq r_{2},\|x-\Phi\| \leq r_{1}\right\}$. From the above discussion,

$$
\begin{aligned}
& \left\|T_{1} y-\Phi\right\| \leq\left[r_{2}+\|\Psi\|\right]^{\tau} \sum_{i=1}^{\omega} A_{i} \Xi_{i}\|\Psi\|^{\tau_{i}-\tau} \leq r_{1}, \\
& \left\|T_{2} x-\Psi\right\| \leq\left[r_{1}+\|\Phi\|\right]^{\delta} \sum_{i=1}^{\omega} B_{i} \bar{\Xi}_{i}\|\Phi\|^{\delta_{i}-\delta} \leq r_{2} .
\end{aligned}
$$

Hence $T(x, y) \in \Omega_{r_{1}, r_{2}}$. By Schauder's fixed point theorem, $T$ has at least one fixed point $(x, y) \in \Omega_{r_{1}, r_{2}}$. Then $(x, y)$ is a solution of $\operatorname{BVP}(1)$.

The proof of Theorem 3.1 is complete.

Theorem 3.2. Suppose that both (B3) and (B4) hold, and there exists $M>0$ such that

$$
\begin{aligned}
f\left(t, t^{\beta-2} x, t^{\beta-3} y, t^{\beta-4} z, t^{\beta-n-2} w\right) \mid & \leq M, \\
g\left(t, t^{\alpha-2} x, t^{\alpha-3} y, t^{\alpha-4} z, t^{\alpha-m-2} w\right) \mid & \leq M
\end{aligned}
$$

for all $t \in(0,1)$ and $x, y, z, w \in \mathbb{R}$. Then $\mathrm{BVP}(1)$ has at least one positive solution. 
Proof. In Theorem 3.1, choose $\psi(t)=\phi(t)=0$ and $\omega=1, \tau_{1 j}=\delta_{1 j}=0$ $(j=1,2,3,4), A_{1}=B_{1}=M$.

Now, we list the following assumptions:

(B7) $\psi \in L^{1}(0,1)$ and there exists $N_{0}>0$ such that $|\psi(t)| \leq N_{0}$ for all $t \in(0,1)$.

(B8) $\phi \in L^{1}(0,1)$ and there exists $M_{0}>0$ such that $|\phi(t)| \leq M_{0}$ for all $t \in(0,1)$.

(B9) $f:(0,1) \times \mathbb{R}^{4} \rightarrow[0, \infty)$ is continuous and there exist $\tau_{1}, \tau_{2}, \tau_{3}, \tau_{4}$ $\geq 0$ and $A_{i} \geq 0(i=1,2,3,4)$ such that

$$
\begin{aligned}
\left|f\left(t, t^{\beta-2} x, t^{\beta-3} y, t^{\beta-4} z, t^{\beta-n-2} w\right)-\psi(t)\right| & \\
& \leq A_{1}|x|^{\tau_{1}}+A_{2}|y|^{\tau_{2}}+A_{3}|z|^{\tau_{3}}+A_{4}|w|^{\tau_{4}}
\end{aligned}
$$

for all $t \in(0,1)$ and $x, y, z, w \in \mathbb{R}$.

(B10) $g:(0,1) \times \mathbb{R}^{4} \rightarrow[0, \infty)$ is continuous and there exist $\delta_{1}, \delta_{2}, \delta_{3}, \delta_{4}$ $\geq 0$ and $B_{i} \geq 0(i=1,2,3,4)$ such that

$$
\begin{aligned}
\mid g\left(t, t^{\alpha-2} x, t^{\alpha-3} y, t^{\alpha-4} x,\right. & \left.t^{\alpha-m-2} y\right)-\phi(t) \mid \\
& \leq B_{1}|x|^{\delta_{1}}+B_{2}|y|^{\delta_{2}}+B_{3}|z|^{\delta_{3}}+B_{4}|w|^{\delta_{4}}
\end{aligned}
$$

for all $t \in(0,1)$ and $x, y, z, w \in \mathbb{R}$.

Denote $\tau=\max \left\{\tau_{i}: i=1,2,3,4\right\}$ and $\delta=\max \left\{\delta_{i}: i=1,2,3,4\right\}$ and

$$
\begin{aligned}
\Psi(t)= & \int_{0}^{t} \frac{(t-s)^{\beta-1}}{\Gamma(\beta)} \psi(s) d s-\left[\frac{t^{\beta-1}}{\Gamma(\beta)}-\frac{t^{\beta-2}}{\Gamma(\beta-1)}\right] \int_{0}^{1} \psi(s) d s \\
& -\frac{t^{\beta-2}}{\Gamma(\beta-1)} \int_{0}^{1}(1-s) \psi(s) d s, \\
\Phi(t)= & \int_{0}^{t} \frac{(t-s)^{\alpha-1}}{\Gamma(\alpha)} \phi(s) d s-\left[\frac{t^{\alpha-1}}{\Gamma(\alpha)}-\frac{t^{\alpha-2}}{\Gamma(\alpha-1)}\right] \int_{0}^{1} \phi(s) d s \\
& -\frac{t^{\alpha-2}}{\Gamma(\alpha-1)} \int_{0}^{1}(1-s) \phi(s) d s, \\
P_{i}= & \frac{1}{\Gamma(\alpha+1)}+\frac{\alpha}{\Gamma(\alpha)}+\frac{1}{2 \Gamma(\alpha-1)}=\frac{3 \alpha^{2}-\alpha+2}{2 \Gamma(\alpha+1)}, \\
Q_{i}= & \frac{1}{\Gamma(\alpha)}+\frac{\alpha-1}{\Gamma(\alpha-1)}+\frac{1}{2 \Gamma(\alpha-2)}=\frac{3 \alpha^{2}-7 \alpha+6}{2 \Gamma(\alpha)}, \\
R_{i}= & \frac{1}{\Gamma(\alpha-1)}+\frac{\alpha-2}{\Gamma(\alpha-2)}+\frac{1}{2 \Gamma(\alpha-3)}=\frac{3 \alpha^{2}-13 \alpha+16}{2 \Gamma(\alpha-1)}, \\
W_{i}= & \frac{1}{\Gamma(\alpha-m+1)}+\frac{\alpha-m}{\Gamma(\alpha-m)}+\frac{1}{2 \Gamma(\alpha-m-1)} \\
= & \frac{3 \alpha^{2}-(6 m+1) \alpha+3 m^{2}+m+2}{2 \Gamma(\alpha-m+1)},
\end{aligned}
$$




$$
\Xi=\max \left\{\frac{3 \alpha^{2}-(6 j+1) \alpha+3 j^{2}+j+2}{2 \Gamma(\alpha-j+1)}: j=0,1,2, m\right\},
$$

and

$$
\begin{aligned}
\bar{P}_{i} & =\frac{1}{\Gamma(\beta+1)}+\frac{\beta}{\Gamma(\beta)}+\frac{1}{2 \Gamma(\beta-1)}=\frac{3 \beta^{2}-\beta+2}{2 \Gamma(\beta+1)}, \\
\bar{Q}_{i} & =\frac{1}{\Gamma(\beta)}+\frac{\beta-1}{\Gamma(\beta-1)}+\frac{1}{2 \Gamma(\beta-2)}=\frac{3 \beta^{2}-7 \beta+6}{2 \Gamma(\beta)}, \\
\bar{R}_{i} & =\frac{1}{\Gamma(\beta-1)}+\frac{\beta-2}{\Gamma(\beta-2)}+\frac{1}{2 \Gamma(\beta-3)}=\frac{3 \beta^{2}-13 \beta+16}{2 \Gamma(\beta-1)}, \\
\bar{W}_{i} & =\frac{1}{\Gamma(\beta-n+1)}+\frac{\beta-n}{\Gamma(\beta-n)}+\frac{1}{2 \Gamma(\beta-n-1)} \\
& =\frac{3 \beta^{2}-(6 n+1) \beta+3 n^{2}+n+2}{2 \Gamma(\beta-n+1)}, \quad i=1,2,3,4, \\
\bar{\Xi} & =\max \left\{\frac{3 \beta^{2}-(6 j+1) \beta+3 j^{2}+j+2}{2 \Gamma(\beta-j+1)}: j=0,1, \ldots, m\right\} .
\end{aligned}
$$

THEOREM 3.3. Suppose that (B7), (B8), (B9) and (B10) hold. Then $\mathrm{BVP}(1)$ has at least one positive solution if

(i) $\tau \delta<1$, or

(ii) $\tau \delta=1$ with

$$
\bar{\Xi} \Xi^{1 / \tau} \sum_{i=1}^{4} B_{i}\|\Phi\|^{\delta_{i}-\delta}\left[\sum_{i=1}^{4} A_{i}\|\Psi\|^{\tau_{i}-\tau}\right]^{1 / \tau}<1,
$$

or

(iii) $\tau \delta>1$ with

$$
\bar{\Xi}\left(\frac{\delta \tau}{\delta \tau-1}\right)^{\delta} \sum_{i=1}^{4} B_{i}\|\Phi\|^{\delta_{i}}+\|\Psi\| \leq \frac{\left(\frac{\|\Phi\|}{\delta \tau-1}\right)^{1 / \tau}}{\left[\Xi \sum_{i=1}^{4} A_{i}\|\Psi\|^{\tau_{i}-\tau}\right]^{1 / \tau}}
$$

Proof. Choose $a_{0}=b_{0}=c_{0}=d_{0}=0$. Then (B7) and (B8) imply (B3) and (B4) respectively. Choose $\omega=4, a_{i}=b_{i}=c_{i}=d_{i}=0(i=1,2,3,4)$, and

$$
\begin{array}{ll}
\left(\tau_{11}, \tau_{12}, \tau_{13}, \tau_{14}\right)=\left(\tau_{1}, 0,0,0\right), & \left(\tau_{21}, \tau_{22}, \tau_{23}, \tau_{24}\right)=\left(0, \tau_{2}, 0,0\right), \\
\left(\tau_{31}, \tau_{32}, \tau_{33}, \tau_{34}\right)=\left(0,0, \tau_{3}, 0\right), & \left(\tau_{41}, \tau_{42}, \tau_{43}, \tau_{44}\right)=\left(0,0,0, \tau_{4}\right), \\
\left(\delta_{11}, \delta_{12}, \delta_{13}, \delta_{14}\right)=\left(\delta_{1}, 0,0,0\right), & \left(\delta_{21}, \delta_{22}, \delta_{23}, \delta_{24}\right)=\left(0, \delta_{2}, 0,0\right), \\
\left(\delta_{31}, \delta_{32}, \delta_{33}, \delta_{34}\right)=\left(0,0, \delta_{3}, 0\right), & \left(\delta_{41}, \delta_{42}, \delta_{43}, \delta_{44}\right)=\left(0,0,0, \delta_{4}\right) .
\end{array}
$$

Then (B9) and (B10) imply (B5) and B6) respectively. By Theorem 3.1, we get (i), (ii) and (iii). 
REMARK 3.1. From (B5), (B6), we know that our assumptions on $f, g$ are different from (BA1) and (BA2). When one considers BVP(I), BVP(II) and BVP(III), similar results can be obtained. We omit the details. But we point out that our assumptions on $f$ and $g$ are weaker than (BA1) and (BA2) since $\rho \sigma=\max \left\{\rho_{1}, \rho_{2}\right\} \max \left\{\sigma_{1}, \sigma_{2}\right\}<1$ is weaker than $\max \left\{\rho_{1}, \rho_{2}\right\}<1$ and $\max \left\{\sigma_{1}, \sigma_{2}\right\}<1$.

Acknowledgements. The authors would like to thank the referees and the editors for their careful reading and some useful comments on improving the presentation of this paper.

This research was supported by the Natural Science Foundation of Guangdong province (No. S2011010001900) and the Foundation for Outstanding Talent in Guangdong Higher Education Project.

\section{References}

[1] R. P. Agarwal, M. Benchohra and S. Hamani, A survey on existence results for boundary value problems of nonlinear fractional differential equations and inclusions, Acta Appl. Math. 109 (2010), 973-1033.

[2] R. P. Agarwal and Y. M. Chow, Iterative methods for a fourth order boundary value problem, J. Comput. Appl. Math. 10 (1984), 203-217.

[3] R. P. Agarwal, Y. Liu, D. O'Regan and C. Tian, Positive solutions of two-point boundary value problems for fractional singular differential equations, Differential Equations 48 (2012), 619-629.

[4] B. Ahmad and A. Alsaedi, Existence and uniqueness of solutions for coupled systems of higher-order nonlinear fractional differential equations, Fixed Point Theory Appl. 2010, art. ID 364560, 17 pp.

[5] B. Ahmad and J. Nieto, Existence results for a coupled system of nonlinear fractional differential equations with three-point boundary conditions, Comput. Math. Appl. 58 (2009), 1838-1843.

[6] B. Ahmad, J. J. Nieto, A. Alsaedi and M. El-Shahed, A study of nonlinear Langevin equation involving two fractional orders in different intervals, Nonlinear Anal. Real World Appl. 13 (2012), 599-606.

[7] A. Arara, M. Benchohra, N. Hamidi, and J. J. Nieto, Fractional order differential equations on an unbounded domain, Nonlinear Anal. 72 (2010), 580-586.

[8] Z. Bai, On positive solutions of a nonlocal fractional boundary value problem, Nonlinear Anal. 72 (2010), 916-924.

[9] A. H. Bokhari, F. M. Mahomed and F. D. Zaman, Invariant boundary value problems for a fourth-order dynamic Euler-Bernoulli beam equation, J. Math. Phys. 53 (2012), 043703, 6 pp.

[10] R. Dehghani and K. Ghanbari, Triple positive solutions for boundary value problem of a nonlinear fractional differential equation, Bull. Iranian Math. Soc. 33 (2007), $1-14$.

[11] V. Gafiychuk, B. Datsko, V. Meleshko and D. Blackmore, Analysis of the solutions of coupled nonlinear fractional reaction-diffusion equations, Chaos Solitons Fractals 41 (2009), 1095-1104. 
[12] V. D. Gejji, Positive solutions of a system of nonautonomous fractional differential equations, J. Math. Anal. Appl. 320 (2005), 56-64.

[13] C. S. Goodrich, Existence of a positive solution to systems of differential equations of fractional order, Comput. Math. Appl. 62 (2011), 1251-1268.

[14] C. P. Gupta, Existence and uniqueness theorems for the bending of an elastic beam equation, Applicable Anal. 26 (1988), 289-304.

[15] E. R. Kaufmann and N. Kosmatov, Elastic beam problem with higher order derivatives, Nonlinear Anal. Real World Appl. 8 (2007), 811-821.

[16] A. A. Kilbas, H. M. Srivastava and J. J. Trujillo, Theory and Applications of Fractional Differential Equations, North-Holland Math. Stud. 204, Elsevier, Amsterdam, 2006.

[17] A. A. Kilbas and J. J. Trujillo, Differential equations of fractional order: methods, results and problems-I, Applicable Anal. 78 (2001), 153-192.

[18] S. Labidi and N.-E. Tatar, Blow-up of solutions for a nonlinear beam equation with fractional feedback, Nonlinear Anal. 74 (2011), 1402-1409.

[19] M. P. Lazarević, Finite time stability analysis of $P D^{\alpha}$ fractional control of robotic time-delay systems, Mech. Res. Comm. 33 (2006), 269-279.

[20] Y. Liu, Existence and non-existence of positive solutions of BVPs for fractional order elastic beam equations with a non-Carathéodory nonlinearity, Appl. Math. Modelling 38 (2014), 620-640.

[21] Y. Liu, Multiple positive solutions of BVPs for singular fractional differential equations with non-Carathéodory nonlinearities, Math. Modelling Anal. 19 (2014), 395416.

[22] H. Lu, L. Sun and J. Sun, Existence of positive solutions to a non-positive elastic beam equation with both ends fixed, Boundary Value Problems 2012, 2012:56, 10 pp.

[23] R. Ma, Multiple positive solutions for a semipositone fourth-order boundary value problem, Hiroshima Math. J. 33 (2003), 217-227.

[24] N. Nyamoradi and T. Bashiri, Existence of positive solutions for fractional differential systems with multi point boundary conditions, Ann. Univ. Ferrara Sez. VII Sci. Mat. 59 (2013), 375-392.

[25] I. Podlubny, Geometric and physical interpretation of fractional integral and fractional differentiation, Fract. Calc. Appl. Anal. 5 (2002), 367-386; Correction, ibid. 6 (2013), 103-110.

[26] S. Z. Rida, H. M. El-Sherbiny and A. A. M. Arafa, On the solution of the fractional nonlinear Schrödinger equation, Phys. Lett. A 372 (2008), 553-558.

[27] S. G. Samko, A. A. Kilbas and O. I. Marichev, Fractional Integrals and Derivatives. Theory and Applications, Gordon and Breach, 1993.

[28] X. Su, Boundary value problem for a coupled system of nonlinear fractional differential equations, Appl. Math. Lett. 22 (2009), 64-69.

[29] J. R. L. Webb, G. Infante and D. Franco, Positive solutions of nonlinear fourth order boundary value problems with local and nonlocal boundary conditions, Proc. Roy. Soc. Edinburgh 138 (2008), 427-446.

[30] X. Xu, D. Jiang and C. Yuan, Multiple positive solutions for the boundary value problem of a nonlinear fractional differential equation, Nonlinear Anal. 71 (2009), 4676-4688.

[31] Q. Yao, Positive solutions for eigenvalue problems of four-order elastic beam equations, Appl. Math. Lett. 17 (2004), 237-243.

[32] S. Zhang, Existence results of positive solutions to boundary value problem for fractional differential equation, Positivity 13 (2009), 583-599. 
[33] S. Zhang, Positive solution of singular boundary value problem for nonlinear fractional differential equation with nonlinearity that changes sign, Positivity 16 (2012), 177-193.

Yuji Liu, Shengping Chen

Department of Mathematics

Guangdong University of Finance and Economics

Guangzhou 510320, P.R. China

E-mail: liuyuji888@sohu.com 
